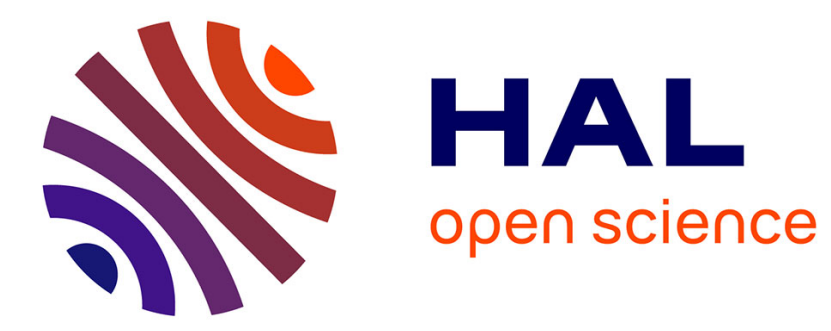

\title{
Stochastic target problems with controlled loss in jump diffusion models
}

\author{
Ludovic Moreau
}

\section{To cite this version:}

Ludovic Moreau. Stochastic target problems with controlled loss in jump diffusion models. SIAM Journal on Control and Optimization, 2011, 49 (6), pp.2577-2607. 10.1137/100802268 . hal-00515522v3

HAL Id: hal-00515522

https://hal.science/hal-00515522v3

Submitted on 17 Aug 2011

HAL is a multi-disciplinary open access archive for the deposit and dissemination of scientific research documents, whether they are published or not. The documents may come from teaching and research institutions in France or abroad, or from public or private research centers.
L'archive ouverte pluridisciplinaire HAL, est destinée au dépôt et à la diffusion de documents scientifiques de niveau recherche, publiés ou non, émanant des établissements d'enseignement et de recherche français ou étrangers, des laboratoires publics ou privés. 


\title{
Stochastic Target Problems with Controlled Loss in Jump Diffusion Models
}

\author{
Ludovic Moreau* \\ CEREMADE, Paris Dauphine University \\ moreau@ceremade.dauphine.fr
}

August 14, 2011

\begin{abstract}
In this paper, we consider a mixed diffusion version of the stochastic target problem introduced in [3]. This consists in finding the minimum initial value of a controlled process which guarantees to reach a controlled stochastic target with a given level of expected loss. It can be converted into a standard stochastic target problem, by increasing both the state space and the dimension of the control. In our mixed-diffusion setting, the main difficulty comes from the presence of jumps, which leads to the introduction of a new kind of controls that take values in an unbounded set of measurable maps. This has non trivial technical impacts on the formulation and derivation of the associated partial differential equations.
\end{abstract}

Key words: Stochastic target problem, mixed diffusion process, discontinuous viscosity solutions, quantile hedging.

\section{Introduction}

A general stochastic target problem with controlled loss can be formulated as follows. For $0 \leq$ $t \leq T$, we are given two controlled diffusion processes $\left\{X_{t, x}^{\nu}(s), t \leq s \leq T\right\}$ and $\left\{Y_{t, x, y}^{\nu}(s), t \leq\right.$ $s \leq T\}$ with values respectively in $\mathbb{R}^{d}$ and $\mathbb{R}$, satisfying the initial condition $\left(X_{t, x}^{\nu}(t), Y_{t, x, y}^{\nu}(t)\right)=$ $(x, y)$. The aim of the controller is to find the minimal initial condition $y$ for which it is possible to find a control $\nu$ satisfying $\mathbb{E}\left[\Psi\left(X_{t, x}^{\nu}(T), Y_{t, x, y}^{\nu}(T)\right)\right] \geq p$ for some given measurable map $\Psi$, non-decreasing in the $y$-variable, and for a level $p$. Namely, he wants to compute:

$$
\widehat{v}(t, x, p):=\inf \left\{y \geq-\kappa: \mathbb{E}\left[\Psi\left(X_{t, x}^{\nu}(T), Y_{t, x, y}^{\nu}(T)\right)\right] \geq p \text { for some control } \nu\right\}
$$

where $\kappa \in \mathbb{R}_{+}$. When $p=1$ and $\Psi(x, y)=\mathbb{1}_{\{V(x, y) \geq 0\}}$ for some map $V$, then

$$
v(t, x):=\widehat{v}(t, x, 1)=\inf \left\{y \geq-\kappa: \mathbb{P}\left[V\left(X_{t, x}^{\nu}(T), Y_{t, x, y}^{\nu}(T)\right) \geq 0\right] \geq 1 \text { for some control } \nu\right\}(1.2
$$

coincides with the stochastic target problem studied in Bouchard [2], and in Soner and Touzi [9] and [10] for Brownian controlled SDEs. In the above mentioned papers, the authors restricted to the setting of controls with values in a compact subset of $\mathbb{R}^{d}$. Their proofs are heavily relying on this assumption.

Only recently, Bouchard, Elie and Touzi [3] considered the case of controls taking values in a possibly unbounded subset of $\mathbb{R}^{d}$. Their main motivation was to study problems of the form (1.1) for $\Psi(x, y)=\mathbb{1}_{\{V(x, y) \geq 0\}}$, as above, but with $0<p<1$, i.e.

\footnotetext{
*The author expresses his gratitude to Bruno Bouchard for leading advices, careful reading and presentation suggestions.
} 


$$
\widehat{v}(t, x, p)=\inf \left\{y \geq-\kappa: \mathbb{P}\left[V\left(X_{t, x}^{\nu}(T), Y_{t, x, y}^{\nu}(T)\right) \geq 0\right] \geq p \text { for some control } \nu\right\} .
$$

Such problems have already been discussed by Föllmer and Leukert [6] in the context of financial mathematics. In this paper, the process $X$ represents the prices of some given securities. The process $Y$ models the wealth of an investor, based on some portfolio strategy $\nu$. Importantly, the coefficients of the diffusion $Y$ are linear in the control variable and the process $X$ is not affected by the control $\nu$. In this context, Föllmer and Leukert [6] used a duality argument to convert this problem into a classical test problem in mathematical statistics. They then obtained a solution by an application of the Neyman Pearson lemma. But the use of the duality argument heavily relies on the linearity of the coefficients of $Y$ in the control variable and on the fact that $X$ is not affected by it. In particular, the duality approach of [6] does not extend to general non linear controlled diffusion cases. This was the initial motivation of Bouchard, Elie and Touzi [3] for proposing a more general treatment of the above problem, at least for Markovian settings.

In order to deal with the problem (1.3), Bouchard, Elie and Touzi [3] introduced an additional controlled diffusion process $P_{t, p}^{\alpha}$, which appears to (essentially) correspond to the conditional probability of reaching the target $V\left(X_{t, x}^{\nu}(T), Y_{t, x, y}^{\nu}(T)\right) \geq 0$. This allowed them to rewrite the problem (1.3) in the form

$$
\widehat{v}(t, x, p)=\inf \left\{y \geq-\kappa: \mathbb{1}_{\left\{V\left(X_{t, x}^{\nu}(T), Y_{t, x, y}^{\nu}(T)\right) \geq 0\right\}} \geq P_{t, p}^{\alpha}(T) \text { for some }(\nu, \alpha)\right\},
$$

where $\alpha$ is a predictable square integrable process coming from the martingale representation $\mathbb{P}\left[V\left(X_{t, x}^{\nu}(T), Y_{t, x, y}^{\nu}(T)\right) \geq 0 \mid \mathcal{F}.\right]=P_{t, p_{0}}^{\alpha}:=p_{0}+\int_{t}^{*} \alpha_{s} \cdot d W_{s}$, for some $p_{0} \geq p$. The key point is that this reformulation reduces the original problem (1.3) into a classical stochastic target problem of the form (1.2), as studied in [9] and [10], for an augmented system $(X, Y, P)$ and an augmented control $(\nu, \alpha)$. The major difference being that the new control $\alpha$ can no longer be assumed to take values in a compact set, as it is given by the martingale representation theorem.

By introducing new arguments, Bouchard, Elie and Touzi [3] were able to provide a PDE characterization for the value function $\widehat{v}$ in the sense of discontinuous viscosity solutions, for a discontinuous operator which corresponds to the one used in [9] and [10] up to a non-trivial relaxation.

The first aim of the present paper is to extend the work of Bouchard, Elie and Touzi [3] to the setting of jump diffusions, as [2] extended [9] and [10] to processes with jumps. We also want to discuss more general problems of the form (1.1).

We follow the key idea of [3] so as to convert the problem $\widehat{v}$ into a singular stochastic target problem by diffusing the conditional expectation $\mathbb{E}\left[\Psi\left(X_{t, x}^{\nu}(T), Y_{t, x, y}^{\nu}(T)\right) \mid \mathcal{F}_{s}\right]$ for $s \in[t, T]$, and considering it as an additional controlled state variable $P_{t, p}^{\alpha, \chi}$, where the additional control $\chi$ comes from the jump part of the martingale representation.

The main new technical difficulty is due to the presence of jumps and of the new control $\chi$. First, it leads to an additional (non-local) term in the PDE characterization, as in [2]. However, part of the control now takes values in an unbounded set of measurable maps, as opposed to a compact subset of $\mathbb{R}^{d}$ in [2]. This leads to a new (non-trivial) relaxation of the non-local part of the associated operator, in comparison to [2] and [3]. Second, because of the presence of this non-local operator, due to the presence of jumps, the discussion of the boundary conditions at $p=0$ and $p=1$ in the context of (1.2) is significantly more difficult than in [3]. Moreover, [3] discussed only problems of the from (1.2), while we shall consider more general problems of the form (1.1), which are only mentioned in [3]. In particular, we shall see that the convex facelifting phenomenon in the $p$-variable observed in [3] for (1.2) extends to a much more general context.

The rest of the paper is organized as follows. Section 2 presents the general formulation of stochastic target problem with unbounded measurable map controls. It contains the statement of the corresponding dynamic programming equation. Section 3 contains the arguments allowing 
us to translate the problem of expected controlled loss into the case of singular stochastic target problem of the previous section. The boundary conditions for the stochastic target problem with controlled expected loss, and the special case of controlled success ratio, are discussed in this section.

In all this paper, elements of $\mathbb{R}^{n}, n \geq 1$, are identified to column vectors, the superscript ${ }^{T}$ stands for transposition, - denotes the scalar product on $\mathbb{R}^{n},|\cdot|$ the Euclidean norm, and $\mathbb{M}^{n}$ denotes the set of $n$-dimensional square matrices. We denote by $\mathbb{S}^{n}$ the subset of elements of $\mathbb{M}^{n}$ which are symmetric. For a subset $\mathcal{O}$ of $\mathbb{R}^{n}, n \geq 1$, we denote by $\overline{\mathcal{O}}$ its closure, by $\operatorname{Int}(\mathcal{O})$ its interior and by $\operatorname{dist}(x, \mathcal{O})$ the Euclidean distance from $x$ to $\mathcal{O}$ with the convention $\operatorname{dist}(x, \emptyset)=\infty$. Finally, we denote by $B_{r}(x)$ the open ball of radius $r>0$ centered at $x \in \mathbb{R}^{n}$. Given a locally bounded map $v$ on a subset $B$ of $\mathbb{R}^{n}$, we define the lower and upper semicontinuous envelopes

$$
v_{*}(b):=\liminf _{B \ni b^{\prime} \rightarrow b} v\left(b^{\prime}\right) \quad v^{*}(b):=\limsup _{B \ni b^{\prime} \rightarrow b} v\left(b^{\prime}\right), b \in \bar{B} .
$$

The convex hull of a function $f$ will be denoted $\odot(f)$, and we recall that it is the greatest convex function lower or equal to $f$. We will use the same notation for the convex hull of a subset, i.e. $\odot(A)$ is the convex hull of the subset $A$, and we recall that it is the smallest convex subset containing $A$, in the sense of inclusion.

In this paper, inequalities between random variable have to be understood in the a.s. sense.

\section{Singular stochastic target problems}

\subsection{Problem formulation}

Let $T>0$ be a fixed time, $E$ a borel subset of $\mathbb{R}_{+}$, equipped with its Borel field $\mathcal{E}, J(d e, d t)=$ $\sum_{i=1}^{d} J^{i}(d e, d t)$ be a $E$-marked right-continuous point process defined on a complete probability space $(\Omega, \mathcal{F}, \mathbb{P})$. Let $W$ be a $\mathbb{R}^{d}$-Brownian motion defined on $(\Omega, \mathcal{F}, \mathbb{P})$, such that $W$ and $J$ are independent. We denote by $\mathbb{F}:=\left\{\mathcal{F}_{t}, 0 \leq t \leq T\right\}$ the $\mathbb{P}$-completed filtration generated by $(W ., J(d e, \cdot))$. We assume that $\mathcal{F}_{0}$ is trivial. The random measure $J(d e, d t)$ is assumed to have a predictable $(\mathbb{P}, \mathbb{F})$-intensity kernel $\lambda(d e) d t$ such that $\lambda(E)<\infty$, and we denote by $\widetilde{J}(d e, d t):=J(d e, d t)-\lambda(d e) d t$ the associated compensated random measure. By $\mathbb{H}_{\lambda}^{2}$, we denote the set of maps $\chi: \Omega \times[0, T] \times E \rightarrow \mathbb{R}$ which are $\mathcal{P} \otimes \mathcal{E}$ measurable $^{1}$ and such that

$$
\|\chi\|_{\mathbb{H}_{\lambda}^{2}}:=\left(\mathbb{E}\left[\int_{0}^{T} \int_{E} \chi_{t}(e)^{2} \lambda(d e) d t\right]\right)^{\frac{1}{2}}<\infty .
$$

As will be clear below, we can always assume that $\mathbb{P}[J(E \backslash \operatorname{supp}(\lambda),[0, T])>0]=0$, and therefore that $E=\operatorname{supp}(\lambda)$.

Let $\mathcal{U}_{0}=\mathcal{U}_{0}^{1} \times \mathcal{U}_{0}^{2}$ be the collection of predictable processes $\nu=\left(\nu^{1}, \nu^{2}\right)$ with $\nu^{1} \in \mathbb{L}^{2}([0, T]) \mathbb{P}_{-}$ a.s., $\nu^{2} \in \mathbb{H}_{\lambda}^{2}$, and with values in a given closed subset $U=U^{1} \times \mathbb{L}_{\lambda}^{2}$ of $\mathbb{R}^{d} \times \mathbb{L}_{\lambda}^{2}$, where $\mathbb{L}_{\lambda}^{2}$ denotes the set of measurable functions $\pi: E \rightarrow \mathbb{R}$ such that $\|\pi\|_{\lambda}^{2}<\infty$, with $\|\pi\|_{\lambda}^{2}:=\int_{E}|\pi(e)|^{2} \lambda(d e)$.

For $t \in[0, T], z=(x, y) \in \mathbb{R}^{d} \times \mathbb{R}$ and $\nu:=\left(\nu^{1}, \nu^{2}\right) \in \mathcal{U}_{0}$, we define $Z_{t, z}^{\nu}:=\left(X_{t, x}^{\nu}, Y_{t, x, y}^{\nu}\right)$ as the $\mathbb{R}^{d} \times \mathbb{R}$-valued solution of the stochastic differential equation

$$
\begin{aligned}
& d X(s)=\mu_{X}\left(X(s), \nu_{s}\right) d s+\sigma_{X}\left(X(s), \nu_{s}\right) d W_{s}+\int_{E} \beta_{X}\left(X(s-), \nu_{s}^{1}, \nu_{s}^{2}(e), e\right) J(d e, d s) \\
& d Y(s)=\mu_{Y}\left(Z(s), \nu_{s}\right) d s+\sigma_{Y}\left(Z(s), \nu_{s}\right) d W_{s}+\int_{E} \beta_{Y}\left(Z(s-), \nu_{s}^{1}, \nu_{s}^{2}(e), e\right) J(d e, d s)
\end{aligned}
$$

satisfying the initial condition $Z(t)=(x, y)$. Here,

\footnotetext{
${ }^{1} \mathcal{P}$ denotes the $\sigma$-algebra of $\mathbb{F}$-predictable subsets of $\Omega \times[0, T]$.
} 


$$
\begin{aligned}
& \left(\mu_{X}, \sigma_{X}\right): \mathbb{R}^{d} \times U \rightarrow \mathbb{R}^{d} \times \mathbb{M}^{d} \\
& \left(\mu_{Y}, \sigma_{Y}\right): \mathbb{R}^{d} \times \mathbb{R} \times U \rightarrow \mathbb{R} \times \mathbb{R}^{d}
\end{aligned}
$$

are locally Lipschitz, and are assumed to satisfy, for $u:=\left(u^{1}, u^{2}\right) \in U=U^{1} \times \mathbb{L}_{\lambda}^{2}$,

$$
\left|\mu_{Y}(x, y, u)\right|+\left|\mu_{X}(x, u)\right|+\left|\sigma_{Y}(x, y, u)\right|+\left|\sigma_{X}(x, u)\right| \leq K(x, y)\left(1+\left|u^{1}\right|+\left\|u^{2}\right\|_{\lambda}\right)
$$

where $K$ is a locally bounded map. Moreover

$$
\begin{array}{r}
\beta_{X}: \mathbb{R}^{d} \times U \times E \rightarrow \mathbb{R}^{d} \\
\beta_{Y}: \mathbb{R}^{d} \times \mathbb{R} \times U \times E \rightarrow \mathbb{R}
\end{array}
$$

are continuous and are assumed to satisfy, for some $M \geq 0$,

$$
\begin{gathered}
\int_{E}\left(\left|\beta_{X}(x, u(e), e)\right|^{2}+\left|\beta_{Y}(z, u(e), e)\right|^{2}\right) \lambda(d e) \leq M\left(1+|z|^{2}+\left|u^{1}\right|^{2}+\left\|u^{2}\right\|_{\lambda}^{2}\right) \\
\int_{E}\left|\beta_{X}(x, u(e), e)-\beta_{X}\left(x^{\prime}, u(e), e\right)\right|^{2} \lambda(d e) \leq M\left|x-x^{\prime}\right|^{2} \\
\int_{E}\left|\beta_{Y}(z, u(e), e)-\beta_{Y}\left(z^{\prime}, u(e), e\right)\right|^{2} \lambda(d e) \leq M\left|z-z^{\prime}\right|^{2},
\end{gathered}
$$

where we use the notation $u(e)=\left(u^{1}, u^{2}(e)\right)$. We denote by $\mathcal{U}=\mathcal{U}^{1} \times \mathcal{U}^{2}$ a subset of elements of $\mathcal{U}_{0}$ for which (2.1) admits an unique strong solution for all given initial data. We assume furthermore that any constant controls with values in $U$ belongs to $\mathcal{U}$. We also allow for state constraints and we denote by $\mathbf{X}$ the interior of the support of the controlled process $X$.

Let $V$ be a measurable map from $\mathbb{R}^{d+1}$ to $\mathbb{R}$ such that, for every fixed $x$, the function

$$
y \longmapsto V(x, y) \text { is non-decreasing and right continuous. }
$$

We then define the stochastic target problem as follows

$$
v(t, x):=\inf \left\{y \geq-\kappa: V\left(X_{t, x}^{\nu}(T), Y_{t, x, y}^{\nu}(T)\right) \geq 0 \text { for some } \nu \in \mathcal{U}\right\},
$$

with $\kappa \in \mathbb{R}_{+} \cup\{+\infty\}$. At this point, the set $U$ may not be bounded, and we will see later that dealing with unbounded controls will be required in the analysis of Section 3 .

In order to be consistent and avoid the process $Y$ to cross the level $-\kappa$, we shall assume all over this paper that

$$
\begin{aligned}
\mu_{Y}(x,-\kappa, u) \geq 0, \quad \sigma_{Y}(x,-\kappa, u)=0 \quad \text { and } \quad \beta_{Y}(x, y, u, e) \geq-(y+\kappa) \\
\\
\text { for all }(x, y, u, e) \in \mathbf{X} \times \mathbb{R} \times U \times E .
\end{aligned}
$$

As in [2], our analysis requires that

$$
y^{\prime} \geq y \text { and } y \in \Gamma(t, x) \Rightarrow y^{\prime} \in \Gamma(t, x) \quad \text { for all } \quad\left(t, x, y, y^{\prime}\right) \in[0, T] \times \mathbb{R}^{d} \times \mathbb{R} \times \mathbb{R}
$$

where

$$
\Gamma(t, x):=\left\{y \geq-\kappa: V\left(X_{t, x}^{\nu}(T), Y_{t, x, y}^{\nu}(T)\right) \geq 0 \text { for some } \nu \in \mathcal{U}\right\} .
$$

This allows to characterize the closure of $\Gamma(t, x)$ as $[v(t, x),+\infty)$, which will be of important use in the following.

Remark 2.1. Let us observe that this problem can be formulated equivalently as

$$
v(t, x):=\inf \left\{y \geq-\kappa: Y_{t, x, y}^{\nu}(T) \geq g\left(X_{t, x}^{\nu}(T)\right) \text { for some } \nu \in \mathcal{U}\right\},
$$

where $g$ is the generalized inverse of $V$ at 0 :

$$
g(x):=\inf \{y \geq-\kappa: V(x, y) \geq 0\}
$$

recall (2.3). 
Example 2.1. Consider the case where $\mathbf{X}=(0, \infty)^{d}$ and $X$ is defined by the stochastic differential equation

$$
\begin{gathered}
d X_{t, x}(s)=\mu\left(X_{t, x}(s)\right) d s+\sigma\left(X_{t, x}(s)\right) d W_{s}+\int_{E} \beta\left(X_{t, x}(s-), e\right) J(d e, d s) \\
X_{t, x}(t)=x \in(0, \infty)^{d},
\end{gathered}
$$

with $Y_{t, x, y}^{\nu}$ given by

$$
Y_{t, x, y}^{\nu}(s)=y+\int_{t}^{s} \nu_{r}^{1} \cdot d X_{t, x}(r), \quad \text { for } \quad s \geq t \quad \text { and } \quad \nu=\left(\nu^{1}, \nu^{2}\right) \in \mathcal{U} .
$$

This corresponds to the situation where the process $X$ is not affected by the control:

$$
\mu_{X}(x, u)=\mu(x), \quad \sigma_{X}(x, u)=\sigma(x) \quad \text { and } \quad \beta_{X}(x, u(e), e)=\beta(x, e) \quad \text { are independent of } u
$$

and

$$
\mu_{Y}(x, y, u):=u^{1} \cdot \mu(x), \quad \sigma_{Y}(x, y, u):=\sigma^{T}(x) u^{1}, \quad \beta_{Y}(x, y, u(e), e):=u^{1} \cdot \beta(x, e) .
$$

In financial mathematics, the process $X$ should be interpreted as the price of $d$ risky securities. Because of the jump diffusions, we are in an incomplete market, so that the uniqueness of a $\mathbb{P}$-equivalent martingale measure is not satisfied. The process $Y$ should be interpreted as the wealth process induced by the trading strategy $\nu$, where $\nu_{s}^{1}$ indicates the number of units of the assets in the portfolio at time $s$.

Finally, for

$$
V(x, y):=y-g(x) \text { for some Lipschitz continuous function } g: \mathbb{R} \rightarrow \mathbb{R}_{+},
$$

$v(t, x)$ coincides with the usual superhedging price of the contingent claim $g\left(X_{t, x}(T)\right)$.

\subsection{Main results}

The main result of this section is the derivation of the dynamic programming equation corresponding to the stochastic target problem (2.2), in the present context of possibly unbounded controls and jumps.

Before to state our main results, we need to introduce additional notations. Given a smooth function $\varphi, u \in U$ and $e \in E$, we now define the operators

$$
\begin{aligned}
& \mathcal{L}^{u} \varphi(t, x):=\partial_{t} \varphi(t, x)+\mu_{X}(x, u) \cdot D \varphi(t, x)+\frac{1}{2} \operatorname{Trace}\left(\sigma_{X} \sigma_{X}^{T}(x, u) D^{2} \varphi(t, x)\right) \\
& \mathcal{G}^{u, e} \varphi(t, x):=\beta_{Y}(x, \varphi(t, x), u(e), e)-\varphi\left(t, x+\beta_{X}(x, u(e), e)\right)+\varphi(t, x),
\end{aligned}
$$

where $\partial_{t} \varphi$ stands for the partial derivative with respect to $t, D \varphi$ and $D^{2} \varphi$ denote the gradient vector and the Hessian matrix with respect to the $x$ variable.

We then define the following relaxed semi-limits

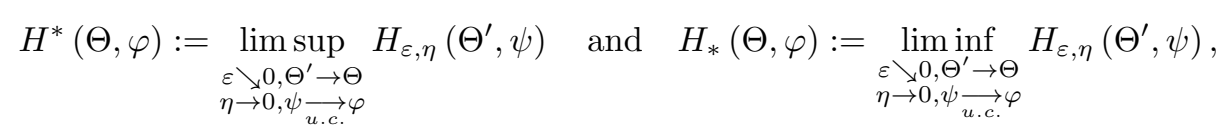

where, for $\Theta=(t, x, y, k, q, A) \in \mathbb{R}_{+} \times \mathbb{R}^{d} \times \mathbb{R} \times \mathbb{R} \times \mathbb{R}^{d} \times \mathbb{S}^{d}, \psi \in \mathcal{C}^{1,2}\left([0, T] \times \mathbb{R}^{d}\right), \varepsilon \geq 0$ and $\eta \in[-1,1]$,

$$
H_{\varepsilon, \eta}(\Theta, \psi):=\sup _{u \in \mathcal{N}_{\varepsilon, \eta}(t, x, y, q, \psi)} \mathbf{A}^{u}(\Theta)
$$

with 


$$
\begin{gathered}
\mathbf{A}^{u}(\Theta):=\mu_{Y}(x, y, u)-k-\mu_{X}(x, u) \cdot q-\frac{1}{2} \operatorname{Trace}\left[\sigma_{X} \sigma_{X}^{T}(x, u) A\right], \\
\mathcal{N}_{\varepsilon, \eta}(t, x, y, q, \psi):=\left\{u \in U:\left|N^{u}(x, y, q)\right| \leq \varepsilon \text { and } \Delta^{u, e}(t, x, y, \psi) \geq \eta \quad \text { for } \lambda \text {-a.e. } e \in E\right\}, \\
N^{u}(x, y, q):=\sigma_{Y}(x, y, u)-\sigma_{X}(x, u)^{T} q \\
\Delta^{u, e}(t, x, y, \psi):=\beta_{Y}(x, y, u(e), e)-\psi\left(t, x+\beta_{X}(x, u(e), e)\right)+y
\end{gathered}
$$

and the convergence $\psi \underset{\text { u.c. }}{\longrightarrow} \varphi$ in (2.5) has to be understood in the sense that $\psi$ converges uniformly on compact subsets towards $\varphi$.

Remark 2.2. Note that the operator $H^{*}$ would not be upper-semicontinuous in $\varphi$, for the u.c. convergence, without the relaxation in the test function on the non-local part. This is the counterpart of the local relaxation introduced in [3] on the derivatives of the test function.

Also notice that, given $\eta \in[-1,1],\left(\mathcal{N}_{\varepsilon, \eta}\right)_{\varepsilon \geq 0}$ is non-decreasing in $\varepsilon$ so that

$$
H_{*}(\Theta, \varphi):=\liminf _{\substack{\eta \rightarrow 0, \Theta^{\prime} \rightarrow \Theta \\ \psi \underset{u . c .}{\longrightarrow} \varphi}} H_{0, \eta}\left(\Theta^{\prime}, \psi\right) .
$$

For ease of notations, we shall often simply write $H v(t, x)$ in place of $H\left(t, x, v(t, x), \partial_{t} v(t, x)\right.$, $\left.D v(t, x), D^{2} v(t, x), v\right)$. We shall similarly use the notations $H^{*} v$ and $H_{*} v$.

As in [3], [9] and [10], the proof of the subsolution property requires an additional regularity assumption on the set valued map $\mathcal{N}_{0, \eta}(\cdot, f)$.

Assumption 2.1. (Continuity of $\left.\mathcal{N}_{0, \eta}(t, x, y, q, f)\right)$ For $f \in \mathcal{C}^{0}\left([0, T] \times \mathbb{R}^{d}\right), \eta>0$, let $B$ be a subset of $[0, T) \times \boldsymbol{X} \times \mathbb{R} \times \mathbb{R}^{d}$ such that $\mathcal{N}_{0, \eta}(\cdot, f) \neq \emptyset$ on $B$. Then, for every $\varepsilon>$ $0,\left(t_{0}, x_{0}, y_{0}, q_{0}\right) \in \operatorname{Int}(B)$, and $u_{0} \in \mathcal{N}_{0, \eta}\left(t_{0}, x_{0}, y_{0}, q_{0}, f\right)$, there exists an open neighborhood $B^{\prime}$ of $\left(t_{0}, x_{0}, y_{0}, q_{0}\right)$ and a locally Lipschitz map $\bar{\nu}$ defined on $B^{\prime}$ such that $\left|\bar{\nu}\left(t_{0}, x_{0}, y_{0}, q_{0}\right)-u_{0}\right| \leq \varepsilon$ and $\bar{\nu}(t, x, y, q) \in \mathcal{N}_{0, \eta}(t, x, y, q, f)$ on $B^{\prime}$.

We also assume that $v$ is locally bounded, so that $v_{*}$ and $v^{*}$ are finite. Our first result characterizes $v$ as a discontinuous viscosity solution of the variational inequation (2.13) in the following sense.

Theorem 2.1. The function $v_{*}$ is a viscosity supersolution on $[0, T) \times \boldsymbol{X}$ of

$$
H^{*} v_{*} \geq 0
$$

If in addition Assumption 2.1 holds, then the function $v^{*}$ is a viscosity subsolution on $[0, T) \times \boldsymbol{X}$ of

$$
\min \left\{H_{*} v^{*}, v^{*}+\kappa\right\} \leq 0
$$

The proof of this result is reported in Section 2.3.

Example 2.2. In the context of Example 2.1, direct computations show that $v_{*}$ is a viscosity supersolution on $[0, T) \times(0, \infty)^{d}$ of

$$
0 \leq \min \left\{-\partial_{t} \varphi-\frac{1}{2} \sigma^{2} D^{2} \varphi, D \varphi \cdot \beta(\cdot, e)-\varphi(\cdot+\beta(\cdot, e))+\varphi\right\} \text {, for } \lambda \text {-a.e. } e \in E
$$

and that $v^{*}$ is a viscosity subsolution of

$$
0 \geq \min \left\{-\partial_{t} \varphi-\frac{1}{2} \sigma^{2} D^{2} \varphi, D \varphi \cdot \beta(\cdot, e)-\varphi(\cdot+\beta(\cdot, e))+\varphi\right\} \text { for } e \in E^{\prime} \in \mathcal{E} \text { s.t. } \lambda\left(E^{\prime}\right)>0
$$


We next discuss the boundary conditions on $\{T\} \times \mathbf{X}$. By the definition of the stochastic target problem, we have

$$
v(T, x)=g(x) \text { for every } x \in \mathbb{R}^{d},
$$

where $g$ is defined in (2.4). However, the possible discontinuities of $v$ might imply that the limits $v_{*}(T, \cdot)$ and $v^{*}(T, \cdot)$ do not agree with this boundary condition. In order to discuss this boundary condition, we need to introduce, as in [3], the set-valued map

$$
\begin{aligned}
\mathbf{N}(t, x, y, q, \psi):=\left\{(r, s) \in \mathbb{R}^{d} \times \mathbb{R}: \exists u \in U\right. & \text { s.t. } r=N^{u}(x, y, q) \\
& \text { and } \left.s \leq \Delta^{u, e}(t, x, y, \psi) \quad \text { for } \lambda \text {-a.e. } e \in E\right\},
\end{aligned}
$$

together with the signed distance function from its complement $\mathbf{N}^{c}$ to the origin:

$$
\delta:=\operatorname{dist}\left(0, \mathbf{N}^{c}\right)-\operatorname{dist}(0, \mathbf{N}),
$$

where we recall that dist stands for the (unsigned) Euclidean distance. Then,

$$
0 \in \operatorname{int}(\mathbf{N}(t, x, y, q, \psi)) \text { iff } \delta(t, x, y, q, \psi)>0 .
$$

The upper and lower-semicontinuous envelopes of $\delta$ are respectively denoted by $\delta^{*}$ and $\delta_{*}$, and we will abuse notation by writing $\delta_{*} v(t, x)=\delta_{*}(t, x, v(t, x), D v(t, x), v)$ and $\delta^{*} v(t, x)=$ $\delta^{*}(t, x, v(t, x), D v(t, x), v)$.

For $\varphi \in \mathcal{C}^{2}\left(\mathbb{R}^{d}\right)$, we similarly define $\delta_{*} \varphi(x)=\delta_{*}(T, x, \varphi(x), D \varphi(x), \varphi)$ and the same definition holds for $\delta^{*} \varphi(x)$.

Remark 2.3. From the convention $\sup \emptyset=-\infty$ and the supersolution property (2.6) in Theorem 2.1, it follows that

$$
\delta^{*} v_{*} \geq 0 \text { on }[0, T) \times \mathbb{R}^{d}
$$

in the viscosity sense. Then, if $\mathbf{N}^{c} \neq \emptyset$, this means that $v$ is subject to a gradient constraint.

Remark 2.4. 1. Assume that for every $(x, y, q)$ and $r \in \mathbb{R}^{d}$, there is an unique solution $\bar{u}(x, y, q, r)$ to the equation $N^{u}(x, y, q)=r$, i.e.

$$
N^{u}(x, y, q)=r \quad \text { iff } \quad u=\bar{u}(x, y, q, r) .
$$

Assume further that $\bar{u}$ is locally Lipschitz continuous, so that Assumption 2.1 trivially holds. For ease of notations, we set $\bar{u}_{0}(x, y, q):=\bar{u}(x, y, q, 0)$. For a bounded set of controls $U$, it follows that, for any smooth function $\varphi, H^{*} \varphi(t, x) \geq 0$ implies that

$$
\begin{gathered}
\bar{u}_{0}(x, \varphi(t, x), D \varphi(t, x)) \in U, \quad \mathbf{A}^{\bar{u}_{0}}\left(\cdot, \varphi, \partial_{t} \varphi, D \varphi, D^{2} \varphi\right)(t, x) \geq 0 \\
\quad \text { and } \Delta^{\bar{u}_{0}, e}(t, x, \varphi(t, x), \varphi) \geq 0 \quad \text { for } \lambda \text {-a.e. } e \in E .
\end{gathered}
$$

Similarly, $H_{*} \varphi(t, x) \leq 0$ implies that

$$
\begin{gathered}
\text { either } \bar{u}_{0}(x, \varphi(t, x), D \varphi(t, x)) \notin \operatorname{int} U, \quad \text { or } \quad \mathbf{A}^{\bar{u}_{0}}\left(\cdot, \varphi, \partial_{t} \varphi, D \varphi, D^{2} \varphi\right)(t, x) \leq 0 \\
\text { or } \Delta^{\bar{u}_{0}, e}(t, x, \varphi(t, x), \varphi)<0 \quad \text { for } \quad e \in E^{\prime} \in \mathcal{E} \quad \text { s.t. } \quad \lambda\left(E^{\prime}\right)>0 .
\end{gathered}
$$

The following result states that the constraint discussed in Remark 2.3 propagates up to the boundary. Here, the main difficulty is due to the unboundedness of the set $U$ and the presence of jumps in the diffusions.

Theorem 2.2. The function $x \mapsto v_{*}(T, x)$ is a viscosity supersolution of

$$
\min \left\{\left(v_{*}(T, \cdot)-g_{*}\right) \mathbb{1}_{\left\{H^{*} v_{*}(T, \cdot)<\infty\right\}}, \delta^{*} v_{*}(T, \cdot)\right\} \geq 0 \text { on } \boldsymbol{X},
$$

and, under Assumption 2.1, $x \in \boldsymbol{X} \mapsto v^{*}(T, x)$ is a viscosity subsolution of

$$
\min \left\{v^{*}(T, \cdot)-g^{*}, \delta_{*} v^{*}(T, \cdot)\right\} \leq 0 \text { on } \boldsymbol{X} \text {. }
$$


We conclude this section by a remark that will be of important use in the proofs of Section 3.5 below.

Remark 2.5. Assume that

$$
\text { ess } \sup _{u \in \mathcal{N} ., e \in E}\left\{\left|\beta_{X}(\cdot, u(e), e)\right|+\left|\beta_{Y}(\cdot, u(e), e)\right|\right\} \text { is locally bounded, and } E \text { is compact. }
$$

Then, the operator $H$ is continuous for the uniform convergence on compact sets in its $\psi \in \mathcal{C}^{1,2}$ parameter.

In this case, the test function $\psi$ appearing in the form $\psi\left(t, x+\beta_{X}(x, u(e), e)\right)$ in the definition of $H^{*}$ can be replaced by $v_{*}$ itself.

To see this, note that for any $\varepsilon>0,\left(t_{0}, x_{0}\right)$ and $\varphi \in \mathcal{C}^{1,2}$ such that $\left(v_{*}-\varphi\right)$ achieves a strict minimum at $\left(t_{0}, x_{0}\right)$, one can find a sequence of smooth function $\varphi_{n}^{\varepsilon}$ such that $\varphi_{n}^{\varepsilon}=\varphi$ on $B_{\varepsilon}\left(t_{0}, x_{0}\right), \varphi_{n}^{\varepsilon} \leq v_{*}$, and $\varphi_{n}^{\varepsilon} \uparrow v_{*}$ uniformly on compact sets of $\left(B_{2 \varepsilon}\left(t_{0}, x_{0}\right)\right)^{c}$.

This allows to replace the original test function $\varphi$ by $v_{*}$ on $\left(B_{2 \varepsilon}\left(t_{0}, x_{0}\right)\right)^{c}$. It then suffices to send $\varepsilon \rightarrow 0$ and use the continuity induced by (2.11).

The same remark holds for the subsolution property.

Remark 2.6. When the set $U$ is bounded, and $\beta_{X} \equiv \beta_{Y} \equiv 0$, i.e. there is no jumps, it was proved in Soner and Touzi [10] that the value function $v$ is a discontinuous viscosity solution of

$$
\sup \left\{\mu_{Y}(x, v(t, x), u)-\mathcal{L}^{u} v(t, x): u \in \mathcal{N}_{0}(x, v(t, x), D v(t, x))\right\}=0,
$$

where

$$
\mathcal{N}_{0}(x, y, q):=\left\{u \in U: N^{u}(x, y, q)=0\right\} \quad \text { and } \quad N^{u}(x, y, q):=\sigma_{Y}(x, y, u)-\sigma_{X}(x, u)^{T} q,
$$

with the standard convention $\sup \emptyset=-\infty$. In the case of a convex compact set $U$, with jumps and $\mathbb{R}^{d}$-valued controls, i.e. $\mathcal{U}^{2}=\{0\}$, Bouchard [2] showed that $v$ is a viscosity solution of an equation of the form

$$
\sup \left\{\min \left\{\mathcal{L}^{u} \varphi(t, x), \inf _{e \in E} \mathcal{G}^{u, e} \varphi(t, x)\right\}: u \in \mathcal{N}_{0}(x, v(t, x), D v(t, x))\right\}=0 .
$$

Finally the case of unbounded set $U$ with no jumps was considered by Bouchard, Elie and Touzi [3]. In this paper, the authors introduced a relaxation on the operator (2.12), in order to deal with this unboundedness. This relaxation applies to the space variable $x$, the function $\varphi$, its gradient and its Hessian matrix, at the local point $(t, x)$. Such a relaxation is required in order to ensure that the sub-solution (resp. super-solution) property is stated in terms of a lower semi-continuous (resp. upper semi-continuous) operator. In our jump-diffusion framework, a similar relaxation is required, but it should involve the additional non-local term $\mathcal{G}^{u, e}$ in (2.13).

\subsection{Derivation of the PDE for singular stochastic target problems}

This section is dedicated to the proof of Theorems 2.1 and 2.2. We first recall the geometric dynamic programming principle of Soner and Touzi [9], see also Bouchard and Vu [4]. We next report the proof of the supersolution properties in Sections 2.3.1 and 2.3.2, and the proof of the subsolution properties in Sections 2.3.3 and 2.3.4.

Theorem 2.3. (Geometric Dynamic Programming Principle) Fix $(t, x) \in[0, T) \times \boldsymbol{X}$ and let $\left\{\theta^{\nu}, \nu \in \mathcal{U}\right\}$ be a family of $[t, T]$-valued stopping times. Then,

(GDP1) If $y>v(t, x)$, then there exists $\nu \in \mathcal{U}$

$$
Y_{t, x, y}^{\nu}\left(\theta^{\nu}\right) \geq v\left(\theta^{\nu}, X_{t, x}^{\nu}\left(\theta^{\nu}\right)\right) .
$$

(GDP2) For every $-\kappa \leq y<v(t, x), \nu \in \mathcal{U}$,

$$
\mathbb{P}\left[Y_{t, x, y}^{\nu}\left(\theta^{\nu}\right)>v\left(\theta^{\nu}, X_{t, x}^{\nu}\left(\theta^{\nu}\right)\right)\right]<1
$$




\subsubsection{The supersolution property on $[0, T) \times \mathbf{X}$}

We follow the arguments of [3] up to non trivial modifications due to the presence of the jumps.

$1^{\text {st }}$ Step: Let $\left(t_{0}, x_{0}\right) \in[0, T) \times \mathbf{X}$ and $\varphi$ be a smooth function such that

$$
\min _{[0, T) \times \mathbf{X}}(\text { strict })\left(v_{*}-\varphi\right)=\left(v_{*}-\varphi\right)\left(t_{0}, x_{0}\right)=0 .
$$

Assume that $H^{*} \varphi\left(t_{0}, x_{0}\right)=:-2 \eta<0$ for some $\eta>0$, and let us work towards a contradiction. Set $\widetilde{\varphi}(t, x):=\varphi(t, x)-\iota\left|x-x_{0}\right|^{4}$ for $\iota>0$. By definition of the upper-semicontinuous operator $H^{*}$ and the fact that $\widetilde{\varphi} \underset{u . c .}{\longrightarrow} \varphi$ as $\iota \rightarrow 0$, we may find $\varepsilon>0$ and $\iota>0$ small enough such that, after possibly changing $\eta>0$

$$
\begin{gathered}
\mu_{Y}(x, y, u)-\mathcal{L}^{u} \widetilde{\varphi}(t, x) \leq-\eta \\
\text { for all } u \in \mathcal{N}_{\varepsilon,-\eta}(t, x, y, D \widetilde{\varphi}(t, x), \widetilde{\varphi}) \text { and }(t, x, y) \in[0, T) \times \mathbf{X} \times \mathbb{R} \text { s.t. } \\
(t, x) \in B_{\varepsilon}\left(t_{0}, x_{0}\right) \text { and }|y-\widetilde{\varphi}(t, x)| \leq \varepsilon
\end{gathered}
$$

where we recall that $B_{\varepsilon}\left(t_{0}, x_{0}\right)$ denotes the ball of radius $\varepsilon$ around $\left(t_{0}, x_{0}\right)$. Notice that we still have

$$
0=v_{*}\left(t_{0}, x_{0}\right)-\widetilde{\varphi}\left(t_{0}, x_{0}\right)=\min _{[0, T) \times \mathbf{X}}(\text { strict })\left(v_{*}-\widetilde{\varphi}\right) .
$$

Let $\partial_{p} B_{\varepsilon}\left(t_{0}, x_{0}\right):=\left\{t_{0}+\varepsilon\right\} \times \bar{B}_{\varepsilon}\left(t_{0}, x_{0}\right) \cup\left[t_{0}, t_{0}+\varepsilon\right) \times \partial B_{\varepsilon}\left(x_{0}\right)$ denote the parabolic boundary of $B_{\varepsilon}\left(t_{0}, x_{0}\right)$. Set $\zeta:=\min _{\partial_{p} B_{\varepsilon}\left(t_{0}, x_{0}\right)}\left(v_{*}-\widetilde{\varphi}\right)$, and observe that $\zeta>0$ since the above minimum is strict. We now define $\mathcal{V}_{\varepsilon}\left(t_{0}, x_{0}\right):=\partial_{p} B_{\varepsilon}\left(t_{0}, x_{0}\right) \cup\left[t_{0}, t_{0}+\varepsilon\right) \times B_{\varepsilon}^{c}\left(x_{0}\right)$, and observe that

$$
\left(v_{*}-\widetilde{\varphi}\right)(t, x) \geq \zeta \wedge \iota \varepsilon^{4}=: \xi>0 \text { for }(t, x) \in \mathcal{V}_{\varepsilon}\left(t_{0}, x_{0}\right)
$$

since $\left(t_{0}, x_{0}\right)$ is a strict minimizer, and $\iota\left|x-x_{0}\right|^{4} \geq \iota \times \varepsilon^{4}$ on $B_{\varepsilon}^{c}\left(x_{0}\right)$.

$2^{\text {nd }}$ step: Let $\left(t_{n}, x_{n}\right)_{n \geq 1}$ be a sequence in $[0, T) \times \mathbf{X}$ which converges to $\left(t_{0}, x_{0}\right)$ and such that $\overline{v\left(t_{n}, x_{n}\right)} \rightarrow v_{*}\left(t_{0}, x_{0}\right)$. Set $y_{n}:=v\left(t_{n}, x_{n}\right)+n^{-1}$ and observe that

$$
\gamma_{n}:=y_{n}-\widetilde{\varphi}\left(t_{n}, x_{n}\right) \rightarrow 0 .
$$

For each $n \geq 1$, we have $y_{n}>v\left(t_{n}, x_{n}\right)$. Thus, it follows from (GDP1) that there exists some $\nu^{n} \in \mathcal{U}$ such that

$$
Y^{n}\left(t \wedge \theta_{n}\right) \geq v\left(t \wedge \theta_{n}, X^{n}\left(t \wedge \theta_{n}\right)\right), \quad t \geq t_{n}
$$

where

$$
\begin{aligned}
& \theta_{n}^{o}:=\left\{s \geq t_{n}:\left(s, X^{n}(s)\right) \notin B_{\varepsilon}\left(t_{0}, x_{0}\right)\right\} \\
& \theta_{n}:=\left\{s \geq t_{n}:\left|Y^{n}(s)-\widetilde{\varphi}\left(s, X^{n}(s)\right)\right| \geq \varepsilon\right\} \wedge \theta_{n}^{o},
\end{aligned}
$$

and

$$
Z^{n}:=\left(X^{n}, Y^{n}\right):=\left(X_{t_{n}, x_{n}}^{\nu^{n}}, Y_{t_{n}, x_{n}, y_{n}}^{\nu^{n}}\right) .
$$

By the inequalities $v \geq v_{*} \geq \widetilde{\varphi}$, this implies that

$$
\begin{aligned}
& Y^{n}\left(t \wedge \theta_{n}\right)-\widetilde{\varphi}\left(t \wedge \theta_{n}, X^{n}\left(t \wedge \theta_{n}\right)\right) \\
& \geq\left[Y^{n}\left(t \wedge \theta_{n}\right)-\widetilde{\varphi}\left(t \wedge \theta_{n}, X^{n}\left(t \wedge \theta_{n}\right)\right)\right] \mathbb{1}_{\left\{t \geq \theta_{n}\right\}} \\
& \geq\left[\left(Y^{n}\left(t \wedge \theta_{n}\right)-\widetilde{\varphi}\left(t \wedge \theta_{n}, X^{n}\left(t \wedge \theta_{n}\right)\right)\right) \mathbb{1}_{\left\{\theta_{n}<\theta_{n}^{\circ}\right\}}\right. \\
& \left.\quad+\left(v_{*}\left(t \wedge \theta_{n}, X^{n}\left(t \wedge \theta_{n}\right)\right)-\widetilde{\varphi}\left(t \wedge \theta_{n}, X^{n}\left(t \wedge \theta_{n}\right)\right)\right) \mathbb{1}_{\left\{\theta_{n}=\theta_{n}^{o}\right\}}\right] \mathbb{1}_{\left\{t \geq \theta_{n}\right\}} \\
& \geq\left[\varepsilon \mathbb{1}_{\left\{\theta_{n}<\theta_{n}^{\circ}\right\}}+\xi \mathbb{1}_{\left\{\theta_{n}=\theta_{n}^{\circ}\right\}}\right] \mathbb{1}_{\left\{t \geq \theta_{n}\right\}}
\end{aligned}
$$

and therefore 


$$
Y^{n}\left(t \wedge \theta_{n}\right)-\widetilde{\varphi}\left(t \wedge \theta_{n}, X^{n}\left(t \wedge \theta_{n}\right)\right) \geq(\varepsilon \wedge \xi) \mathbb{1}_{\left\{t \geq \theta_{n}\right\}} \geq 0 .
$$

$3^{\text {rd }}$ step: Since $\widetilde{\varphi}$ is smooth, it follows from Itô's lemma, (2.15), the definition of $Y^{n}$ and (2.16), that

$$
S_{t}^{n}:=a_{n}+\int_{t_{n}}^{t \wedge \theta_{n}}\left(b_{s}^{n}+d_{s}^{n}\right) d s+\int_{t_{n}}^{t \wedge \theta_{n}} \psi_{s}^{n} d W_{s}+\int_{t_{n}}^{t \wedge \theta_{n}} \int_{E} c_{s}^{n, e} \widetilde{J}(d e, d s) \geq-(\varepsilon \wedge \xi) \mathbb{1}_{\left\{t<\theta_{n}\right\}}
$$

where we recall that $\widetilde{J}$ is the compensated jump measure and

$$
\begin{array}{rlrl}
a_{n} & :=-(\varepsilon \wedge \xi)+\gamma_{n}, & b_{s}^{n}:=\mu_{Y}\left(Z_{s}^{n}, \nu_{s}^{n}\right)-\mathcal{L}^{\nu_{s}^{n}} \widetilde{\varphi}\left(s, X_{s}^{n}\right) \\
c_{s}^{n, e} & :=\beta_{Y}\left(X_{s-}^{n}, Y_{s-}^{n}, \nu_{s}^{n}(e), e\right)-\widetilde{\varphi}\left(s, X_{s-}^{n}+\beta_{X}\left(X_{s-}^{n}, \nu_{s}^{n}(e), e\right)\right)+\widetilde{\varphi}\left(s, X_{s-}^{n}\right) \\
\psi_{s}^{n} & :=N^{\nu^{n}}\left(Z_{s}^{n}, D \widetilde{\varphi}\left(s, X_{s}^{n}\right)\right), & d_{s}^{n}:=\int_{E} c_{s}^{n, e} \lambda(d e) .
\end{array}
$$

In view of (2.15), we have

$$
a_{n} \rightarrow-(\varepsilon \wedge \xi)<0 \text { for } n \rightarrow \infty
$$

$4^{\text {th }}$ step: Let us define:

$$
\begin{aligned}
& A_{1}^{n}:=\left\{s \in\left[t_{n}, \theta_{n}\right]: \min \left\{b_{s}^{n}, c_{s}^{n, e}\right\}>-\eta \text { for } \lambda \text {-a.e. } e \in E\right\} \\
& A_{2}^{n}:=\left\{s \in\left(A_{1}^{n}\right)^{c}: c_{s}^{n, e} \leq-\eta \text { for } e \in E^{\prime} \in \mathcal{E} \text { s.t. } \lambda\left(E^{\prime}\right)>0\right\} \\
& A_{3}^{n}:=\left(A_{1}^{n} \cup A_{2}^{n}\right)^{c}=\left\{s \in\left(A_{1}^{n}\right)^{c}: b_{s}^{n} \leq-\eta \text { and } c_{s}^{n, e}>-\eta \text { for } \lambda \text {-a.e. } e \in E\right\} \\
& A_{4}^{n}:=\left\{(s, e) \in\left(A_{1}^{n}\right)^{c} \times E: c_{s}^{n, e} \leq-\eta / 2\right\} .
\end{aligned}
$$

Observe that (2.14) implies that the process $\psi^{n}$ satisfies:

$$
\left|\psi_{s}^{n}\right|>\varepsilon \text { for } s \in A_{1}^{n} .
$$

We then define, for all $t_{n} \leq t \leq T$,

$$
L_{.}^{n}:=\mathcal{E}\left(\int_{t_{n}} \alpha_{s}^{n} d W_{s}+\int_{t_{n}} \int_{E} \delta_{s}^{n, e} \widetilde{J}(d e, d s)\right)_{\cdot \wedge \theta_{n}}
$$

where $\mathcal{E}$ denotes the Doleans-Dade exponential, and

$$
\begin{aligned}
\alpha_{s}^{n} & :=-\frac{\left(b_{s}^{n}+d_{s}^{n}\right)}{\left|\psi_{s}^{n}\right|^{2}} \psi_{s}^{n} \mathbb{1}_{A_{1}^{n}}(s) \\
\delta_{s}^{n, e} & :=-\mathbb{1}_{\left(A_{1}^{n}\right)^{c}}(s)\left(1-\frac{\eta}{2\left(\left|d_{s}^{n}\right|+1\right)}\right)+\mathbb{1}_{A_{2}^{n}}(s) \mathbb{1}_{A_{4}^{n}}(s, e) \times \frac{2\left(b_{s}^{n}\right)^{+}+\eta}{\eta M_{s}^{n}}
\end{aligned}
$$

where $(x)^{+}=\max (x, 0)$ and

$$
M_{s}^{n}:=\int_{E} \mathbb{1}_{A_{4}^{n}}(s, e) \lambda(d e)
$$

It follows from the very definition of $A_{2}^{n}$ that $M_{s}^{n}>0$ for all $s \in A_{2}^{n}$ and for all $n \geq 1$. We deduce then from our assumptions on the coefficients, (2.20), and the definition of the set of admissible controls $\mathcal{U}$, that $L^{n}$ is well defined as an exponential non-negative local martingale, and is therefore a supermartingale.

We now apply Itô's lemma and use (2.17), to obtain 


$$
\begin{aligned}
L_{t \wedge \theta_{n}}^{n} S_{t \wedge \theta_{n}}^{n}=a_{n} & +\int_{t_{n}}^{t \wedge \theta_{n}} L_{s}^{n}\left\{b_{s}^{n}+d_{s}^{n}+\psi_{s}^{n} \alpha_{s}^{n}+\int_{E} c_{s}^{n, e} \delta_{s}^{n, e} \lambda(d e)\right\} d s \\
& +\int_{t_{n}}^{t \wedge \theta_{n}} L_{s}^{n}\left\{\psi_{s}^{n}+S_{s}^{n} \alpha_{s}^{n}\right\} d W_{s} \\
& +\int_{t_{n}}^{t \wedge \theta_{n}} \int_{E} L_{s}^{n}\left\{c_{s}^{n, e}+S_{s}^{n} \delta_{s}^{n, e}+c_{s}^{n, e} \delta_{s}^{n, e}\right\} \widetilde{J}(d e, d s) \\
& \geq-(\varepsilon \wedge \xi) \mathbb{1}_{\left\{t<\theta_{n}\right\}} L_{t \wedge \theta_{n}}^{n}
\end{aligned}
$$

where, by definitions of $\alpha^{n}$ and $\delta^{n}$,

$$
\begin{aligned}
L_{t \wedge \theta_{n}}^{n} S_{t \wedge \theta_{n}}^{n}=a_{n} & +\int_{t_{n}}^{t \wedge \theta_{n}} L_{s}^{n}\left\{b_{s}^{n}+d_{s}^{n}-\left(b_{s}^{n}+d_{s}^{n}\right) \mathbb{1}_{A_{1}^{n}}(s)\right\} d s \\
& +\int_{E} c_{s}^{n, e} L_{s}^{n}\left[-\mathbb{1}_{\left(A_{1}^{n}\right)^{c}}(s)\left(1-\frac{\eta}{2\left(\left|d_{s}^{n}\right|+1\right)}\right)\right. \\
& \left.\quad+\mathbb{1}_{A_{2}^{n}}(s) \mathbb{1}_{A_{4}^{n}}(s, e) \times \frac{2\left(b_{s}^{n}\right)^{+}+\eta}{\eta M_{s}^{n}}\right] \lambda(d e) d s \\
& +\int_{t_{n}}^{t \wedge \theta_{n}} L_{s}^{n}\left\{\psi_{s}^{n}+S_{s}^{n} \alpha_{s}^{n}\right\} d W_{s} \\
& +\int_{t_{n}}^{t \wedge \theta_{n}} \int_{E} L_{s}^{n}\left\{c_{s}^{n, e}+S_{s}^{n} \delta_{s}^{n, e}+c_{s}^{n, e} \delta_{s}^{n, e}\right\} \widetilde{J}(d e, d s) .
\end{aligned}
$$

Recalling the definition of $d^{n}$ in (2.18), this implies

$$
\begin{aligned}
L_{t \wedge \theta_{n}}^{n} S_{t \wedge \theta_{n}}^{n}=a_{n} & +\int_{t_{n}}^{t \wedge \theta_{n}} L_{s}^{n}\left\{\left(b_{s}^{n}+d_{s}^{n}\right) \mathbb{1}_{\left(A_{1}^{n}\right)^{c}}(s)-\mathbb{1}_{\left(A_{1}^{n}\right)^{c}}(s) d_{s}^{n}\right. \\
& \left.+\mathbb{1}_{\left(A_{1}^{n}\right)^{c}}(s) \frac{\eta d_{s}^{n}}{2\left(\left|d_{s}^{n}\right|+1\right)}\right\} d s \\
& +\int_{E} c_{s}^{n, e} L_{s}^{n}\left[\mathbb{1}_{A_{2}^{n}}(s) \mathbb{1}_{A_{4}^{n}}(s, e) \times \frac{2\left(b_{s}^{n}\right)^{+}+\eta}{\eta M_{s}^{n}}\right] \lambda(d e) d s \\
& +\int_{t_{n}}^{t \wedge \theta_{n}} L_{s}^{n}\left\{\psi_{s}^{n}+S_{s}^{n} \alpha_{s}^{n}\right\} d W_{s} \\
& +\int_{t_{n}}^{t \wedge \theta_{n}} \int_{E} L_{s}^{n}\left\{c_{s}^{n, e}+S_{s}^{n} \delta_{s}^{n, e}+c_{s}^{n, e} \delta_{s}^{n, e}\right\} \widetilde{J}(d e, d s) .
\end{aligned}
$$

Observing that $\left(A_{1}^{n}\right)^{c}=A_{2}^{n} \cup A_{3}^{n}$ and $A_{2}^{n} \cap A_{3}^{n}=\emptyset$, this leads to

$$
\begin{aligned}
L_{t \wedge \theta_{n}}^{n} S_{t \wedge \theta_{n}}^{n}=a_{n}+\int_{t_{n}}^{t \wedge \theta_{n}} L_{s}^{n} & \left\{\left(b_{s}^{n}+\frac{\eta d_{s}^{n}}{2\left(\left|d_{s}^{n}\right|+1\right)}\right) \mathbb{1}_{A_{3}^{n}}(s)\right. \\
& +\left(b_{s}^{n}+\frac{\eta d_{s}^{n}}{2\left(\left|d_{s}^{n}\right|+1\right)}\right) \mathbb{1}_{A_{2}^{n}}(s) \\
& \left.+\int_{E} c_{s}^{n, e}\left[\mathbb{1}_{A_{2}^{n}}(s) \mathbb{1}_{A_{4}^{n}}(s, e) \times \frac{2\left(b_{s}^{n}\right)^{+}+\eta}{\eta M_{s}^{n}}\right] \lambda(d e)\right\} d s \\
& +\int_{t_{n}}^{t \wedge \theta_{n}} L_{s}^{n}\left\{\psi_{s}^{n}+S_{s}^{n} \alpha_{s}^{n}\right\} d W_{s} \\
& +\int_{t_{n}}^{t \wedge \theta_{n}} \int_{E} L_{s}^{n}\left\{c_{s}^{n, e}+S_{s}^{n} \delta_{s}^{n, e}+c_{s}^{n, e} \delta_{s}^{n, e}\right\} \widetilde{J}(d e, d s) .
\end{aligned}
$$

By using the inequalities $c_{s}^{n, e} \leq-\frac{\eta}{2}$ on $A_{4}^{n, e}, b_{s}^{n} \leq-\eta$ on $A_{3}^{n}, \frac{\eta d_{s}^{n}}{2\left(\left|d_{s}^{n}\right|+1\right)} \leq \frac{\eta}{2}$, and the definition of $M_{s}^{n}>0$, we then obtain

$$
\left(b_{s}^{n}+\frac{\eta d_{s}^{n}}{2\left(\left|d_{s}^{n}\right|+1\right)}\right) \mathbb{1}_{A_{3}^{n}}(s) \leq\left(-\eta+\frac{\eta}{2}\right) \mathbb{1}_{A_{3}^{n}}(s)=-\frac{\eta}{2} \mathbb{1}_{A_{3}^{n}}(s) \leq 0
$$


and

$$
\begin{aligned}
\mathbb{1}_{A_{2}^{n}}(s) & \left(b_{s}^{n}+\frac{\eta d_{s}^{n}}{2\left(\left|d_{s}^{n}\right|+1\right)}+\frac{2\left(b_{s}^{n}\right)^{+}+\eta}{\eta M_{s}^{n}} \int_{E} c_{s}^{n, e} \mathbb{1}_{A_{4}^{n}(s, e)} \lambda(d e)\right) \\
& \leq \mathbb{1}_{A_{2}^{n}}(s)\left(b_{s}^{n}+\frac{\eta}{2}-\frac{2\left(b_{s}^{n}\right)^{+}+\eta}{\eta M_{s}^{n}} \frac{\eta}{2} M_{s}^{n}\right) \\
& \leq \mathbb{1}_{A_{2}^{n}}(s)\left(b_{s}^{n}+\frac{\eta}{2}-\left(b_{s}^{n}\right)^{+}-\frac{\eta}{2}\right)=-\mathbb{1}_{A_{2}^{n}}(s)\left(b_{s}^{n}\right)^{-} \leq 0
\end{aligned}
$$

where $x^{-}=\max (-x, 0)$. Thus, (2.22) implies that $L^{n} S^{n}$ is a local supermartingale, which, by $(2.21)$, is bounded from below by the submartingale $-(\varepsilon \wedge \xi) L^{n}$ on $\left[t_{n}, \theta_{n}\right]$. Hence, $L^{n} S^{n}$ is a supermartingale, and it follows from (2.21) again that

$$
0=-(\varepsilon \wedge \xi) \mathbb{E}\left[\mathbb{1}_{\left\{\theta_{n}<\theta_{n}\right\}} L_{\theta_{n}}^{n}\right] \leq \mathbb{E}\left[L_{\theta_{n}}^{n} S_{\theta_{n}}^{n}\right] \leq L_{t_{n}}^{n} S_{t_{n}}^{n}=S_{t_{n}}^{n}=a_{n}
$$

which contradicts (2.19) for $n$ large enough.

Remark 2.7. Note that, in the above proof, the relaxation of the non-local part of the operator in term of u.c. convergence is required in order to pass from the initial test function $\varphi$ to the penalized one $\widetilde{\varphi}$. It allows to obtain the inequality $v_{*} \geq \widetilde{\varphi}+\xi$ outside of the ball $B_{\varepsilon}\left(x_{0}\right)$, which is crucial in our proof. This is not required in [3] where processes are continuous. It is neither required in [2], where the non-local operator is already continuous and the size of the jump is locally bounded.

\subsubsection{The supersolution property on $\{T\} \times \mathbf{X}$}

We split the proof in different lemmas.

Lemma 2.1. Let $x_{0} \in \boldsymbol{X}$ and $\varphi \in \mathcal{C}^{2}(\boldsymbol{X})$ be such that

$$
0=\left(v_{*}(T, \cdot)-\varphi\right)\left(x_{0}\right)=\min _{\boldsymbol{X}}(\operatorname{strict})\left(v_{*}(T, \cdot)-\varphi\right)
$$

then

$$
\delta^{*} \varphi\left(x_{0}\right) \geq 0
$$

Since $\delta^{*}$ is upper semi-continuous, the result follows from exactly the same arguments as in lemma 5.2 in [10]. We therefore omit it.

Lemma 2.2. $v_{*}$ is a viscosity supersolution of

$$
\left(v_{*}(T, \cdot)-g_{*}\right) \mathbb{1}_{\left\{H^{*} v_{*}(T, \cdot)<\infty\right\}} \geq 0 \text { on } \boldsymbol{X} .
$$

Proof. Let $x_{0} \in \mathbf{X}$ and $\varphi$ be a smooth function such that

$$
\min _{\mathbf{X}}(\text { strict })\left(v_{*}(T, \cdot)-\varphi\right)=\left(v_{*}(T, \cdot)-\varphi\right)\left(x_{0}\right) .
$$

$11^{\text {st }}$ step: Assume that $H^{*} v_{*}\left(T, x_{0}\right)<\infty, \varphi\left(x_{0}\right)=v_{*}\left(T, x_{0}\right)<g_{*}\left(x_{0}\right)$, and let us work towards a contradiction. Since $v(T, \cdot)=g$ by the definition of the problem and $g \geq g_{*}$, there is a constant $\eta>0$ such that $\varphi-v(T, \cdot) \leq \varphi-g_{*} \leq-\eta$ on $B_{\varepsilon}\left(x_{0}\right)$ for some $\varepsilon>0$. Since $x_{0}$ is a strict minimizer, we have $2 \zeta:=\min _{x \in \partial B_{\varepsilon}\left(x_{0}\right)} v_{*}(T, x)-\varphi(x)>0$, and it follows from the lower semi-continuity of $v_{*}$ that there exists $r>0$ such that

$$
\begin{gathered}
v(t, x)-\varphi(x) \geq v_{*}(t, x)-\varphi(x) \geq \zeta>0 \text { for all }(t, x) \in[T-r, T] \times \partial B_{\varepsilon}\left(x_{0}\right), \\
v(t, x)-\varphi(x) \geq \zeta \wedge \eta>0 \text { for }(t, x) \in\left([T-r, T) \times \partial B_{\varepsilon}\left(x_{0}\right)\right) \cup\left(\{T\} \times B_{\varepsilon}\left(x_{0}\right)\right)=: \mathcal{V}_{\varepsilon, r}\left(T, x_{0}\right) .
\end{gathered}
$$

Define $\varphi_{\iota}(x):=\varphi(x)-\iota\left|x-x_{0}\right|^{4}$, for $\iota>0$. As in the proof of Section 2.3.1, we have 


$$
v(t, x)-\varphi_{\iota}(x) \geq \zeta \wedge \eta \wedge \iota \varepsilon^{4}=: 2 \xi>0 \text { for }(t, x) \in\left([T-r, T] \times \bar{B}_{\varepsilon}^{c}\left(x_{0}\right)\right) \cup\left(\{T\} \times B_{\varepsilon}\left(x_{0}\right)\right) .
$$

We now use the fact that $H^{*} \varphi\left(x_{0}\right)=: \frac{C}{2}<\infty$. Set

$$
\widetilde{\varphi}(t, x):=\varphi_{\iota}(x)+(C+\eta)(t-T) \leq \varphi_{\iota}(x) .
$$

Then, after possibly changing $\varepsilon, \eta>0$, and for $r, \iota>0$ sufficiently small,

$$
\begin{gathered}
v(t, x)-\widetilde{\varphi}(t, x) \geq \xi>0 \text { for }(t, x) \in \mathcal{V}_{\varepsilon, r}\left(T, x_{0}\right) \cup[T-r, T] \times \bar{B}_{\varepsilon}^{c}\left(x_{0}\right), \\
\mu_{Y}(x, y, u)-\mathcal{L}^{u} \widetilde{\varphi}(t, x) \leq-\eta \text { for all } u \in \mathcal{N}_{\varepsilon,-\eta}(t, x, y, D \widetilde{\varphi}(t, x), \widetilde{\varphi}) \\
\text { and }(t, x, y) \in[T-r, T] \times \mathbf{X} \times \mathbb{R} \text { s.t. } x \in B_{\varepsilon}\left(x_{0}\right) \text { and }|y-\widetilde{\varphi}(t, x)| \leq \varepsilon .
\end{gathered}
$$

Indeed, $\mu_{Y}(x, y, u)-\mathcal{L}^{u} \widetilde{\varphi}(t, x)=\mu_{Y}(x, y, u)-\mathcal{L}^{u} \varphi_{\iota}(x)-C-\eta \leq-\eta$ as soon as $\mu_{Y}(x, y, u)-$ $\mathcal{L}^{u} \varphi_{\iota}(x) \leq C$, and we have $\mathcal{N}_{\varepsilon,-\eta}\left(t, x, y, D \widetilde{\varphi}(t, x), \varphi_{\iota}\right) \subseteq \mathcal{N}_{\varepsilon,-\eta}(t, x, y, D \widetilde{\varphi}(t, x), \widetilde{\varphi})$.

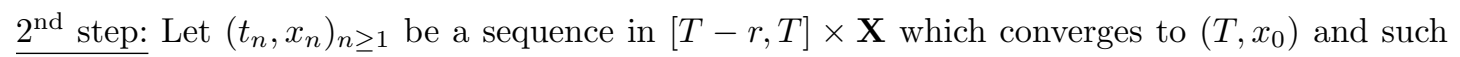
$\left.\overline{\text { that } v\left(t_{n}\right.}, x_{n}\right) \rightarrow v_{*}\left(T, x_{0}\right)$. Set $y_{n}:=v\left(t_{n}, x_{n}\right)+n^{-1}$, and observe that

$$
\gamma_{n}:=y_{n}-\widetilde{\varphi}\left(t_{n}, x_{n}\right) \rightarrow 0 .
$$

For each $n \geq 1$, we have $y_{n}>v\left(t_{n}, x_{n}\right)$. Then, by (GDP1), there exists some $\nu^{n} \in \mathcal{U}$ such that

$$
Y^{n}\left(t \wedge \theta_{n}\right) \geq V\left(t \wedge \theta_{n}, X^{n}\left(t \wedge \theta_{n}\right)\right), \quad t \geq t_{n}
$$

where

$$
\begin{aligned}
& \theta_{n}^{o}:=\left\{s \geq t_{n}:\left(s, X^{n}(s)\right) \notin \mathcal{V}_{\varepsilon, r}\left(T, x_{0}\right)\right\} \\
& \theta_{n}:=\left\{s \geq t_{n}:\left|Y^{n}(s)-\widetilde{\varphi}\left(s, X^{n}(s)\right)\right| \geq \varepsilon\right\} \wedge \theta_{n}^{o},
\end{aligned}
$$

and

$$
Z^{n}:=\left(X^{n}, Y^{n}\right):=\left(X_{t_{n}, x_{n}}^{\nu^{n}}, Y_{t_{n}, x_{n}, y_{n}}^{\nu^{n}}\right) .
$$

Using the inequalities $v \geq v_{*} \geq \widetilde{\varphi}$, this implies that

$$
\begin{aligned}
Y^{n}\left(t \wedge \theta_{n}\right)-\widetilde{\varphi}( & \left.t \wedge \theta_{n}, X^{n}\left(t \wedge \theta_{n}\right)\right) \\
\geq & {\left[Y^{n}\left(t \wedge \theta_{n}\right)-\widetilde{\varphi}\left(t \wedge \theta_{n}, X^{n}\left(t \wedge \theta_{n}\right)\right)\right] \mathbb{1}_{\left\{t \geq \theta_{n}\right\}} } \\
\geq & \mathbb{1}_{\left\{t \geq \theta_{n}\right\}}\left[\left(Y^{n}\left(t \wedge \theta_{n}\right)-\widetilde{\varphi}\left(t \wedge \theta_{n}, X^{n}\left(t \wedge \theta_{n}\right)\right)\right) \mathbb{1}_{\left\{\theta_{n}<\theta_{n}^{\circ}\right\}}\right. \\
& \left.+\left(v\left(t \wedge \theta_{n}, X^{n}\left(t \wedge \theta_{n}\right)\right)-\widetilde{\varphi}\left(t \wedge \theta_{n}, X^{n}\left(t \wedge \theta_{n}\right)\right)\right) \mathbb{1}_{\left\{\theta_{n}=\theta_{n}^{\circ}\right\}}\right] \\
\geq & {\left[\varepsilon \mathbb{1}_{\left\{\theta_{n}<\theta_{n}^{\circ}\right\}}+\xi \mathbb{1}_{\left\{\theta_{n}=\theta_{n}^{\circ}\right\}}\right] \mathbb{1}_{\left\{t \geq \theta_{n}\right\}} }
\end{aligned}
$$

and then

$$
Y^{n}\left(t \wedge \theta_{n}\right)-\widetilde{\varphi}\left(t \wedge \theta_{n}, X^{n}\left(t \wedge \theta_{n}\right)\right) \geq(\varepsilon \wedge \xi) \mathbb{1}_{\left\{t \geq \theta_{n}\right\}} \geq 0 .
$$

By repeating the arguments of steps 3 and 4 of Section 2.3.1, we end up to a contradiction.

\subsubsection{The subsolution property on $[0, T) \times \mathbf{X}$}

The proof of the subsolution property is a straightforward combination of the arguments of [2] and [3]. We provide it for completeness.

$1^{\text {st }}$ step: Let $\left(t_{0}, x_{0}\right) \in[0, T) \times \mathbf{X}$ and $\varphi$ be a smooth function such that

$$
0=\left(v^{*}-\varphi\right)\left(t_{0}, x_{0}\right)>\left(v^{*}-\varphi\right)(t, x) \text { for }\left(t_{0}, x_{0}\right) \neq(t, x) \in[0, T) \times \mathbf{X} .
$$

We assume that $v^{*}\left(t_{0}, x_{0}\right)>-\kappa$ and we show that 


$$
H_{*} \varphi\left(t_{0}, x_{0}\right) \leq 0
$$

Assume to the contrary that

$$
4 \eta:=H_{*} \varphi\left(t_{0}, x_{0}\right)>0 .
$$

By (2.5), and after possibly changing $\eta>0$, we may find $\varepsilon>0$ and $\iota>0$ sufficiently small such that

$$
\mu_{Y}(x, y, u)-\mathcal{L}^{u} \widetilde{\varphi}(t, x) \geq 2 \eta
$$

for some $u \in \mathcal{N}_{0, \eta}(t, x, y, D \widetilde{\varphi}(t, x), \widetilde{\varphi})$, for all $(t, x, y) \in[0, T) \times \mathbf{X} \times \mathbb{R}$ such that $(t, x) \in B_{\varepsilon}\left(t_{0}, x_{0}\right)$ and $|y-\widetilde{\varphi}(t, x)| \leq \varepsilon$, where $\widetilde{\varphi}(t, x):=\varphi(t, x)+\iota\left|x-x_{0}\right|^{4}$. Observe that we still have

$$
0=\left(v^{*}-\widetilde{\varphi}\right)\left(t_{0}, x_{0}\right)=\max _{[0, T) \times \mathbf{X}}(\text { strict })\left(v^{*}-\widetilde{\varphi}\right) .
$$

For $\varepsilon$ sufficiently small, Assumption 2.1 then implies that

$$
\begin{array}{r}
\min \left\{\mu_{Y}(x, y, \widehat{\nu}(t, x, y, D \widetilde{\varphi}(t, x)))-\mathcal{L}^{\widehat{\nu}(t, x, y, D \widetilde{\varphi}(t, x))} \widetilde{\varphi}(t, x),\right. \\
\left.\mathcal{G}^{\widehat{\nu}(t, x, y, D \widetilde{\varphi}(t, x)), e} \widetilde{\varphi}(t, x)\right\} \geq \eta \text { for } \lambda \text {-a.e. } e \in E
\end{array}
$$

for all $(t, x, y) \in[0, T) \times \mathbf{X} \times \mathbb{R}$ s.t. $(t, x) \in B_{\varepsilon}\left(t_{0}, x_{0}\right)$ and $|y-\widetilde{\varphi}(t, x)| \leq \varepsilon$, where $\widehat{\nu}$ is a locally Lipschitz map satisfying

$$
\widehat{\nu}(t, x, y, D \widetilde{\varphi}(t, x)) \in \mathcal{N}_{0, \eta}(t, x, y, D \widetilde{\varphi}(t, x), \widetilde{\varphi}) \text { on } B_{\varepsilon}\left(t_{0}, x_{0}\right) .
$$

Observe that, since $\left(t_{0}, x_{0}\right)$ is a strict maximizer in $(2.24)$, we have

$$
-\zeta:=\max _{\partial_{p} B_{\varepsilon}\left(t_{0}, x_{0}\right)}\left(v^{*}-\widetilde{\varphi}\right)<0
$$

where $\partial_{p} B_{\varepsilon}\left(t_{0}, x_{0}\right)$ denotes the parabolic boundary of $B_{\varepsilon}\left(t_{0}, x_{0}\right)$. Since $\left|x-x_{0}\right|^{4} \geq \varepsilon^{4}$ for $x \notin B_{\varepsilon}\left(x_{0}\right)$

$$
\left(v^{*}-\widetilde{\varphi}\right)(t, x)=\left(v^{*}-\varphi\right)(t, x)-\iota\left|x-x_{0}\right|^{4} \leq-\iota \varepsilon^{4}<0
$$

for $(t, x) \in[0, T) \times B_{\varepsilon}^{c}\left(x_{0}\right)$. Thus, for $(t, x) \in\left(\left[t_{0}, t_{0}+\varepsilon\right) \times B_{\epsilon}^{c}\left(x_{0}\right)\right) \cup\left(\left\{t_{0}+\varepsilon\right\} \times \bar{B}_{\varepsilon}\left(x_{0}\right)\right)$,

$$
\left(v^{*}-\widetilde{\varphi}\right)(t, x) \leq-\left(\iota \varepsilon^{4} \wedge \zeta\right)=:-\xi<0 .
$$

$\underline{2^{\text {nd }}}$ step: We now show that $(2.25),(2.26)$ and (2.27) lead to a contradiction to (GDP2).

Let $\left(t_{n}, x_{n}\right)_{n \geq 1}$ be a sequence in $[0, T) \times \mathbf{X}$ which converges to $\left(t_{0}, x_{0}\right)$ and such that $v\left(t_{n}, x_{n}\right) \rightarrow$ $v^{*}\left(t_{0}, x_{0}\right)$. Set $y_{n}:=v\left(t_{n}, x_{n}\right)-n^{-1}$, and observe that

$$
\gamma_{n}:=y_{n}-\widetilde{\varphi}\left(t_{n}, x_{n}\right) \rightarrow 0
$$

Also notice that $y_{n} \geq-\kappa$ for $n$ large enough.

Let $Z^{n}:=\left(X^{n}, Y^{n}\right)$ denote the solution of (2.1) associated to the Markovian control $\widehat{\nu}^{n}:=$ $\widehat{\nu}\left(\cdot, X^{n}, Y^{n}, D \widetilde{\varphi}\left(\cdot, X^{n}\right)\right)$ and the initial condition $Z^{n}\left(t_{n}\right)=\left(x_{n}, y_{n}\right)$. Since $\widehat{\nu}$ is locally Lipschitz, this solution is well defined up to the stopping time

$$
\theta_{n}:=\inf \left\{s \geq t_{n}:\left|Y^{n}(s)-\widetilde{\varphi}\left(s, X^{n}(s)\right)\right| \geq \varepsilon\right\} \wedge \theta_{n}^{o}
$$

with

$$
\theta_{n}^{o}:=\inf \left\{s \geq t_{n}:\left(s, X^{n}(s)\right) \notin B_{\varepsilon}\left(t_{0}, x_{0}\right)\right\} .
$$

Note that $(2.25),(2.28)$, and a standard comparison theorem implies that 


$$
Y^{n}\left(\theta_{n}\right)-\widetilde{\varphi}\left(\theta_{n}, X^{n}\left(\theta_{n}\right)\right) \geq \varepsilon \quad \text { on } \quad\left\{\left|Y^{n}\left(\theta_{n}\right)-\widetilde{\varphi}\left(\theta_{n}, X^{n}\left(\theta_{n}\right)\right)\right| \geq \varepsilon\right\}
$$

for $n$ large enough. Indeed, $Y^{n}\left(\theta_{n}\right)-\widetilde{\varphi}\left(\theta_{n}, X^{n}\left(\theta_{n}\right)\right) \geq \gamma_{n}>-\varepsilon$ for $n$ large enough. Since $-v \geq-v^{*} \geq-\widetilde{\varphi}$, we then deduce from (2.27), (2.29) and (2.30) that

$$
\begin{aligned}
Y^{n}\left(\theta_{n}\right) & -v\left(\theta_{n}, X^{n}\left(\theta_{n}\right)\right) \\
& \geq \mathbb{1}_{\left\{\theta_{n}<\theta_{n}^{o}\right\}}\left(Y^{n}\left(\theta_{n}\right)-\widetilde{\varphi}\left(\theta_{n}, X^{n}\left(\theta_{n}\right)\right)\right)+\mathbb{1}_{\left\{\theta_{n}=\theta_{n}^{o}\right\}}\left(Y^{n}\left(\theta_{n}^{o}\right)-v^{*}\left(\theta_{n}^{o}, X^{n}\left(\theta_{n}^{o}\right)\right)\right) \\
& \geq \varepsilon \mathbb{1}_{\left\{\theta_{n}<\theta_{n}^{o}\right\}}+\mathbb{1}_{\left\{\theta_{n}=\theta_{n}^{o}\right\}}\left(Y^{n}\left(\theta_{n}^{o}\right)-v^{*}\left(\theta_{n}^{o}, X^{n}\left(\theta_{n}^{o}\right)\right)\right) \\
& \geq \varepsilon \mathbb{1}_{\left\{\theta_{n}<\theta_{n}^{o}\right\}}+\mathbb{1}_{\left\{\theta_{n}=\theta_{n}^{o}\right\}}\left(Y^{n}\left(\theta_{n}^{o}\right)+\xi-\widetilde{\varphi}\left(\theta_{n}^{o}, X^{n}\left(\theta_{n}^{o}\right)\right)\right) \\
& \geq \varepsilon \wedge \xi+\mathbb{1}_{\left\{\theta_{n}=\theta_{n}^{o}\right\}}\left(Y^{n}\left(\theta_{n}^{o}\right)-\widetilde{\varphi}\left(\theta_{n}^{o}, X^{n}\left(\theta_{n}^{o}\right)\right)\right) .
\end{aligned}
$$

We may continue by using Itô's formula:

$$
\begin{aligned}
Y^{n}\left(\theta_{n}\right)-v\left(\theta_{n}, X^{n}\left(\theta_{n}\right)\right) \geq \varepsilon \wedge \xi+\mathbb{1}_{\left\{\theta_{n}=\theta_{n}^{\circ}\right\}} & \left(\gamma_{n}+\int_{t_{n}}^{\theta_{n}} \alpha\left(s, X_{s}^{n}, Y_{s}^{n}\right) d s\right. \\
& \left.+\int_{t_{n}}^{\theta_{n}} \int_{E} \delta\left(s, X_{s}^{n}, Y_{s}^{n}, e\right) J(d e, d s)\right)
\end{aligned}
$$

where

$$
\begin{aligned}
\alpha(t, x, y):=\mu_{Y} & (x, y, \widehat{\nu}(t, x, y, D \widetilde{\varphi}(t, x)))-\mathcal{L}^{\widehat{\nu}(t, x, y, D \widetilde{\varphi}(t, x))} \widetilde{\varphi}(t, x) \\
\delta(t, x, y, e):= & \beta_{Y}(x, y, \widehat{\nu}(t, x, y, D \widetilde{\varphi}(t, x))(e), e) \\
& -\widetilde{\varphi}\left(t, x+\beta_{X}(x, \widehat{\nu}(t, x, y, D \widetilde{\varphi}(t, x))(e), e)\right)+\widetilde{\varphi}(t, x)
\end{aligned}
$$

and the diffusion coefficient vanishes by (2.26). Recalling (2.25), the fact that $\gamma_{n} \rightarrow 0$, and that $\varepsilon, \zeta>0$, this implies that

$$
Y^{n}\left(\theta_{n}\right)>v\left(\theta_{n}, X^{n}\left(\theta_{n}\right)\right) \text { for sufficiently large } n \text {. }
$$

Since the initial position of the process $Y^{n}$ is $y_{n}=v\left(t_{n}, x_{n}\right)-n^{-1}<v\left(t_{n}, x_{n}\right)$, this is clearly in contradiction with (GDP2).

\subsubsection{The subsolution property on $\{T\} \times \mathbf{X}$}

The proof combines arguments used in the two previous sections 2.3.2 and 2.3.3. The only difference between this proof and the one in [3] relies on the presence of the jumps. However, it can be handled by following [2]. We then only explain the main steps. Let $x_{0} \in \mathbf{X}$ and $\varphi$ be a smooth function such that

$$
\max _{\mathbf{X}}(\text { strict })\left(v^{*}(T, \cdot)-\varphi\right)=\left(v^{*}(T, \cdot)-\varphi\right)\left(x_{0}\right)=0 .
$$

Assume that, for some $\eta>0$,

$$
\begin{aligned}
& 0<\delta_{*} \varphi\left(x_{0}\right) \\
& 0<4 \eta<\varphi\left(x_{0}\right)-g^{*}\left(x_{0}\right)=v^{*}\left(T, x_{0}\right)-g^{*}\left(x_{0}\right)
\end{aligned}
$$

Set $\widetilde{\varphi}(t, x)=\varphi(x)+\iota\left(\left|x-x_{0}\right|^{4}+\sqrt{T-t}\right)$. Since the partial derivatives in $x$ of $\varphi$ and $\widetilde{\varphi}$ are the same for $x=x_{0}$, by (2.8) and Assumption 2.1, using the fact that $\widetilde{\varphi} \geq \varphi$, for $\iota>0$ small enough, after possibly changing $\eta>0$, we can find $r, \varepsilon>0$ and a locally Lipschitz map $\widehat{\nu}$ satisfying,

$$
\widehat{\nu}(t, x, y, D \widetilde{\varphi}(t, x)) \in \mathcal{N}_{0, \eta}(t, x, y, D \widetilde{\varphi}(x), \widetilde{\varphi})
$$

such that

$$
\begin{aligned}
& 0<\delta_{*} \widetilde{\varphi}(t, x) \\
& 0<4 \eta<\widetilde{\varphi}\left(T, x_{0}\right)-g^{*}\left(x_{0}\right)=v^{*}\left(T, x_{0}\right)-g^{*}\left(x_{0}\right)
\end{aligned}
$$


for all $(t, x, y) \in[T-r, T) \times \mathbf{X} \times \mathbb{R}$ s.t. $x \in B_{r}\left(x_{0}\right)$ and $|y-\widetilde{\varphi}(t, x)| \leq \varepsilon$. Since $\partial_{t} \widetilde{\varphi} \rightarrow-\infty$ as $t \rightarrow T$, we deduce that, for $r>0$ small enough,

$$
\mu_{Y}(x, y, \widehat{\nu}(t, x, y, D \widetilde{\varphi}(t, x)))-\mathcal{L}^{\widehat{\nu}(t, x, y, D \widetilde{\varphi}(t, x))} \widetilde{\varphi}(t, x) \geq \eta
$$

for all $(t, x, y) \in[T-r, T) \times \mathbf{X} \times \mathbb{R}$ s.t. $x \in B_{r}\left(x_{0}\right)$ and $|y-\widetilde{\varphi}(t, x)| \leq \varepsilon$. Also observe that, since $v^{*}-\widetilde{\varphi}$ is upper-semicontinuous and $\left(v^{*}-\widetilde{\varphi}\right)\left(T, x_{0}\right)=0$, we can choose $r>0$ such that

$$
v(t, x) \leq \widetilde{\varphi}(t, x)+\frac{\varepsilon}{2} \text { for all }(t, x) \in[T-r, T) \times B_{r}\left(x_{0}\right) .
$$

Moreover, combining the identity $v\left(T, x_{0}\right)=g\left(x_{0}\right),(2.33),(2.34),(2.35)$, the fact that $x_{0}$ achieves a strict maximum, and using similar arguments as those of Section 2.3.2 above, we see that

$$
v(t, x)-\widetilde{\varphi} \leq-\left(\zeta \wedge \iota \varepsilon^{4}\right)=:-\xi
$$

for all $(t, x) \in\left([T-r, T] \times \overline{B_{r}^{c}}\left(x_{0}\right)\right) \cup\left(\{T\} \times B_{r}\left(x_{0}\right)\right)$ and for some $r, \varepsilon>0$ small enough, but so that the above inequalities still hold.

By following the arguments in step 2 of Section 2.3.3, we see that $(2.33),(2.32),(2.35)$ and (2.36) lead to a contradiction of (GDP2).

\section{Target reachability with controlled expected loss}

\subsection{Problem reduction}

We now turn to the main motivation for the above analysis: the stochastic target problem with controlled expected loss.

Let $\Psi$ be a measurable map from $\mathbb{R}^{d+1}$ to $\mathbb{R}$ such that, for every fixed $x$, the function

$$
y \longmapsto \Psi(x, y) \text { is non-decreasing and right continuous. }
$$

We define $L$ as the closed convex hull of the image of $\Psi$

$$
L:=\bar{\odot}(\Psi(\mathbf{X} \times[-\kappa, \infty)))=[m, M],
$$

with $m<M, m, M \in[-\infty,+\infty]$.

For $p \in L$, we define the stochastic target problem with controlled expected loss as follows:

$$
\widehat{v}(t, x, p):=\inf \left\{y \geq-\kappa: \mathbb{E}\left[\Psi\left(X_{t, x}^{\nu}(T), Y_{t, x, y}^{\nu}(T)\right)\right] \geq p \text { for some } \nu \in \mathcal{U}\right\},
$$

with $\kappa \in \mathbb{R}_{+} \cup\{+\infty\}$.

The aim of this section is to convert the problem (3.1) into the class of standard stochastic target problems as defined in Section 2. The dynamic programming equation for the target reachability with controlled expected loss will then be deduced directly from Theorem 2.1 above.

Following [3], we introduce an additional controlled state variable

$$
P_{t, p}^{\alpha, \chi}(s):=p+\int_{t}^{s} \alpha_{r} \cdot d W_{r}+\int_{t}^{s} \int_{E} \chi_{s, e} \widetilde{J}(d e, d s), \quad s \in[t, T],
$$

where the additional controls $\alpha, \chi$ are $\mathbb{F}$-predictable measurable processes, with $\chi \in \mathbb{H}_{\lambda}^{2}$ and $\alpha$ is $\mathbb{R}^{d}$-valued and such that $\mathbb{E}\left[\int_{0}^{T}\left|\alpha_{s}\right|^{2} d s\right]<\infty$. We denote by $\mathcal{A}$ the collection of such processes $(\alpha, \chi)$. For $\widehat{\nu}:=(\nu, \alpha, \chi)$, we then set $\widehat{X}^{\widehat{\nu}}:=\left(X^{\nu}, P^{\alpha, \chi}\right)$. We also define $\widehat{\mathbf{X}}:=\mathbf{X} \times L, \widehat{U}:=$ $U \times \mathbb{R}^{d} \times \mathbb{L}_{\lambda}^{2}$, and denote by $\widehat{\mathcal{U}}=\mathcal{U} \times \mathcal{A}$ the corresponding set of admissible controls. Abusing notations, we also set $Y^{\widehat{\nu}}=Y^{\nu}$. Finally, we introduce the function

$$
\widehat{V}(\widehat{x}, y):=\Psi(x, y)-p, \quad \text { for } \quad y \geq-\kappa \quad \text { and } \quad \widehat{x}=(x, p) \in \overline{(\mathbf{X} \times L)} \text {. }
$$


We make the following assumption, which allows us to use the stochastic integral representation theorem.

Assumption 3.1. $\Psi\left(X_{t, x}^{\nu}(T), Y_{t, x, y}^{\nu}(T)\right)$ is square integrable for all initial conditions $(t, x, y) \in$ $[0, T] \times \boldsymbol{X} \times[-\kappa,+\infty)$ and controls $\nu \in \mathcal{U}$.

Following the arguments of [3], we can now relate $\widehat{v}$ to a stochastic target problem with unbounded controls, and controls taking the form of measurable functions on $E$.

Proposition 3.1. For all $t \in[0, T]$ and $\widehat{x}=(x, p) \in \widehat{\boldsymbol{X}}$, we have

$$
\begin{array}{r}
\widehat{v}(t, \widehat{x})=\inf \left\{y \geq-\kappa: \widehat{V}\left(\widehat{X}_{t, \widehat{v}}^{\widehat{\nu}}(T), Y_{t, x, y}^{\widehat{\nu}}(T)\right) \geq 0 \text { for some } \widehat{\nu}=(\nu, \alpha, \chi) \in \widehat{\mathcal{U}}\right\} \\
=\inf \left\{y \geq-\kappa: \widehat{V}\left(\widehat{X}_{t, \widehat{v}}^{\widehat{\nu}}(T), Y_{t, x, y}^{\widehat{\nu}}(T)\right) \geq 0 \text { and } P_{t, p}^{\alpha, \chi} \in L \text { for some } \widehat{\nu}=(\nu, \alpha, \chi) \in \widehat{\mathcal{U}}\right\} .
\end{array}
$$

Proof. We denote by $u(t, x, p)$ and $w(t, x, p)$ the value functions appearing on the right-hand side of (3.2) and (3.3) respectively.

$1^{\text {st }}$ step: We first show that $\widehat{v} \geq u$. For $y>\widehat{v}(t, x, p)$, we may find $\nu \in \mathcal{U}$ such that $p_{0}:=$ $\overline{\mathbb{E}}\left[\Psi\left(X_{t, x}^{\nu}(T), Y_{t, x, y}^{\nu}(T)\right)\right] \geq p$. By the stochastic integral representation theorem, recall Assumption 3.1 , there exists $(\alpha, \chi) \in \mathcal{A}$ such that

$$
\Psi\left(X_{t, x}^{\nu}(T), Y_{t, x, y}^{\nu}(T)\right)=p_{0}+\int_{t}^{T} \alpha_{s} \cdot d W_{s}+\int_{t}^{T} \int_{E} \chi_{s, e} \widetilde{J}(d e, d s)=P_{t, p_{0}}^{\alpha, \chi}(T) .
$$

Since $p_{0} \geq p$, it follows that $\Psi\left(X_{t, x}^{\nu}(T), Y_{t, x, y}^{\nu}(T)\right) \geq P_{t, p}^{\alpha, \chi}(T)$, and therefore $y \geq u(t, x, p)$ from the definition of the problem $u$.

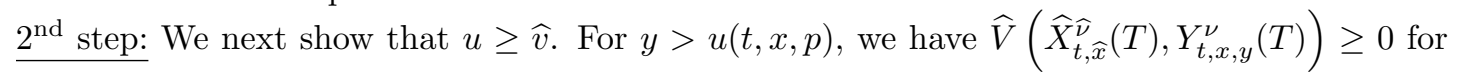
some $\widehat{\nu}=(\nu, \alpha, \chi) \in \widehat{\mathcal{U}}$. It follows that

$$
\mathbb{E}\left[\widehat{V}\left(\widehat{X}_{t, \widehat{x}}^{\widehat{\nu}}(T), Y_{t, x, y}^{\widehat{\nu}}(T)\right)\right]=\mathbb{E}\left[\Psi\left(X_{t, x}^{\nu}(T), Y_{t, x, y}^{\nu}(T)\right)-P_{t, p}^{\alpha, \chi}(T)\right] \geq 0,
$$

and since $P_{t, p}^{\alpha, \chi}$ is a martingale

$$
\mathbb{E}\left[\Psi\left(X_{t, x}^{\nu}(T), Y_{t, x, y}^{\nu}(T)\right)\right] \geq p=\mathbb{E}\left[P_{t, p}^{\alpha, \chi}(T)\right],
$$

so that $y \geq \widehat{v}(t, x, p)$ by the definition of $\widehat{v}$.

$3^{\text {rd }}$ step: The inequality $u \leq w$ is obvious. To see that the converse inequality holds, consider

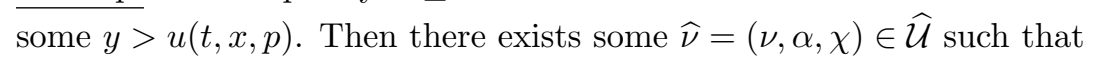

$$
\Psi\left(X_{t, x}^{\nu}(T), Y_{t, x, y}^{\nu}(T)\right) \geq P_{t, p}^{\alpha, \chi}(T) .
$$

Define

$$
\begin{gathered}
\tau:=T \wedge \inf \left\{s>t: P_{t, p}^{\alpha, \chi}(s) \leq m\right\} \text { and } \\
\widetilde{\alpha}_{s}:=\alpha_{s} \mathbb{1}_{\{s \leq \tau\}}, \widetilde{\chi}_{s, e}:=\left[-\left(\chi_{s, e} \vee\left(m-P_{t, p}^{\alpha, \chi}(s-)\right)\right)^{-}+\left(\chi_{s, e}\right)^{+}\right] \mathbb{1}_{\{s \leq \tau\}} \text { for } s \in[t, T] .
\end{gathered}
$$

Clearly, $P_{t, p}^{\alpha, \chi}(T)=P_{t, p}^{\widetilde{\alpha}, \tilde{x}}(T)$ on the event $\{\tau=T\}$. Since $P_{t, p}^{\widetilde{\alpha}, \tilde{\chi}}(T)=m$ on the event $\{\tau<T\}$, it follows from (3.4) that

$$
\Psi\left(X_{t, x}^{\nu}(T), Y_{t, x, y}^{\nu}(T)\right) \geq P_{t, p}^{\widetilde{\alpha}, \tilde{\chi}}(T) .
$$

We finally observe that $P_{t, p}^{\widetilde{\alpha}, \widetilde{\chi}}(T) \geq m$ by the definition of $\widetilde{\alpha}$ and $\widetilde{\chi}$, and that the last inequality implies that $P_{t, p}^{\widetilde{\alpha}, \tilde{\chi}}(T) \leq M$. By the martingale property of the process $P_{t, p}^{\widetilde{\alpha}, \tilde{\chi}}$, we conclude that it is valued in the interval $[m, M]=L$. Hence, $y \geq w(t, x, p)$. 
Let us observe that the problem (3.2) can be alternatively formulated as

$$
\widehat{v}(t, x, p)=\inf \left\{y \geq-\kappa: Y_{t, x, y}^{\widehat{\nu}}(T) \geq \widehat{g}\left(\widehat{X}_{t, \widehat{v}}^{\widehat{v}}(T)\right) \text { for some } \widehat{\nu}=(\nu, \alpha, \chi) \in \widehat{\mathcal{U}}\right\}
$$

where $\widehat{g}$ is the generalized inverse of $\widehat{V}$ at 0

$$
\widehat{g}(\widehat{x}):=\inf \{y: \widehat{V}(\widehat{x}, y) \geq 0\} .
$$

Remark 3.1. 1. In the case where the infimum in the definition of $\widehat{v}(t, x, p)$ is achieved and there exists a control $\nu \in \mathcal{U}$ satisfying $\mathbb{E}\left[\Psi\left(X_{t, x}^{\nu}(T), Y_{t, x, y}^{\nu}(T)\right)\right]=p$ with $y=\widehat{v}(t, x, p)$, the above argument shows that the corresponding process $P_{t, p}^{\alpha, \chi}$ coincides with the conditional expectation of $\Psi\left(X_{t, x}^{\nu}(T), Y_{t, x, y}^{\nu}(T)\right)$, i.e.

$$
P_{t, p}^{\alpha, \chi}(s)=\mathbb{E}\left[\Psi\left(X_{t, x}^{\nu}(T), Y_{t, x, y}^{\nu}(T)\right) \mid \mathcal{F}_{s}\right] \quad \text { for } s \in[t, T] .
$$

2. Equation (3.3) shows that one can restrict to controls $\alpha$ and $\chi$ such that $P_{t, p}^{\alpha, \chi}$ takes values in $L$. This is rather natural since the latter should be interpreted as a conditional expectation of $\Psi$, which convex hull is $L$, and this corresponds to the natural domain $[m, M]$ of the variable $p$. Notice also that the value function $\widehat{v}(\cdot, p)$ is constant for $p<m$, and equal $\infty$ for $p>M$. In both cases, the natural domain of $\widehat{v}$ is therefore $[0, T] \times \overline{\mathbf{X}} \times[m, M]$.

3. Moreover, in the special case where $m$ and/or $M$ are finite, the fact that $P_{t, p}^{\alpha, \chi}$ takes values in $L$ allows us to consider that the jump coefficient $\chi$ is bounded. This will be usefull in the proofs of Section 3.5. Indeed we may write in that particular case

$$
m-P_{t, p}^{\alpha, \chi}(s-) \leq \chi_{s} \leq M-P_{t, p}^{\alpha, \chi}(s-),
$$

with $P_{t, p}^{\alpha, \chi}(s-) \in[m, M]$.

Example 3.1. Given a non-negative function $h$, let us consider the case where $\check{\Psi}(x, y)=\frac{y}{h(x)} \wedge 1$, with the convention $\frac{y}{0}=+\infty$ for $y \in \mathbb{R}_{+}$. For $\kappa=0$, we then obtain

$$
\check{v}(t, x, p)=\inf \left\{y \in \mathbb{R}_{+}: \mathbb{E}\left[\frac{Y_{t, x, y}^{\nu}(T)}{g\left(X_{t, x}^{\nu}(T)\right)} \wedge 1\right] \geq p \text { for some } \nu \in \mathcal{U}\right\},
$$

which is the problem of the expected success ratio studied in [6]. Using (3.2), we see that the above problem reduces to

$$
\check{v}(t, x, p)=\inf \left\{y \in \mathbb{R}_{+}: \check{V}\left(\widehat{X}_{t, x, p}^{\widehat{\nu}}(T), Y_{t, x, y}^{\widehat{\nu}}(T)\right) \geq 0 \text { for some } \widehat{\nu}=(\nu, \alpha, \chi) \in \widehat{\mathcal{U}}\right\},
$$

where $\check{V}(x, p, y)=\check{\Psi}(x, y)-p$.

Example 3.2. One can similarly recover the problem of stochastic target under controlled probability of success studied in [3] and [6]:

$$
\widetilde{v}(t, x, p):=\inf \left\{y \in \mathbb{R}_{+}: \mathbb{P}\left[\widetilde{\Psi}\left(X_{t, x}^{\nu}(T), Y_{t, x, y}^{\nu}\right) \geq 0\right] \geq p \text { for some } \nu \in \mathcal{U}\right\},
$$

for some measurable map $\widetilde{\Psi}$ from $\mathbb{R}^{d+1}$ into $\mathbb{R}$ such that, for every fixed $x \in \mathbb{R}^{d}$, the function $y \mapsto \widetilde{\Psi}(x, y)$ is non-decreasing and right-continuous. The reduction of the problem (3.2) leads to

$$
\widetilde{v}(t, x, p):=\inf \left\{y \in \mathbb{R}_{+}: \widetilde{V}\left(\widehat{X}_{t, x, p}^{\widehat{\nu}}(T), Y_{t, x, y}^{\widehat{\nu}}\right) \geq 0 \text { for some } \widehat{\nu} \in \widehat{\mathcal{U}}\right\},
$$

where $\widetilde{V}(x, p, y)=\mathbb{1}_{\{\widetilde{\Psi}(x, y) \geq 0\}}-p$. 


\subsection{PDE characterization in the domain}

In view of Proposition 3.1, the PDE characterization of Theorem 2.1 can be extended to the problem (3.1). Let us first introduce notations associated to the augmented system.

For $\widehat{u}=(u, \alpha, \pi) \in \widehat{U}$ and $\widehat{x}=(x, p) \in \widehat{\mathbf{X}}$, set

$$
\widehat{\mu}(\widehat{x}, \widehat{u}):=\left(\begin{array}{c}
\mu_{X}(x, u) \\
-\int_{E} \pi(e) \lambda(d e)
\end{array}\right), \quad \widehat{\sigma}(\widehat{x}, \widehat{u}):=\left(\begin{array}{c}
\sigma_{X}(x, u) \\
\alpha^{T}
\end{array}\right), \quad \widehat{\beta}(\widehat{x}, \widehat{u}(e), e):=\left(\begin{array}{c}
\beta_{X}(x, u(e), e) \\
\pi(e)
\end{array}\right) .
$$

Recalling 3. of Remark 3.1, we also introduce, for $(x, k, q, A) \in \mathbb{R} \times \mathbb{R} \times \mathbb{R}^{d+1} \times \mathbb{S}^{d+1}, \widehat{u}=$ $(u, \alpha, \pi) \in \widehat{U}, \varepsilon>0$ and $\eta \in[-1,1]$,

$$
\begin{gathered}
\widehat{N}^{\widehat{u}}(\widehat{x}, y, q):=\sigma_{Y}(x, y, u)-\widehat{\sigma}(\widehat{x}, \widehat{u})^{T} q=N^{u}\left(x, y, q_{x}\right)-q_{p} \alpha, \text { for } q=\left(q_{x}, q_{p}\right) \in \mathbb{R}^{d} \times \mathbb{R} \\
\widehat{\Delta}^{\widehat{u}, e}(t, \widehat{x}, y, \psi):=\beta_{Y}(x, y, u, e)-\psi(t, \widehat{x}+\widehat{\beta}(\widehat{x}, \widehat{u}(e), e))+y \\
\widehat{\mathcal{N}}_{\varepsilon, \eta}(t, \widehat{x}, y, q, \psi):=\left\{\widehat{u} \in \widehat{U}:\left|\widehat{N}^{\widehat{u}}(\widehat{x}, y, q)\right| \leq \varepsilon,\right. \\
\left.p+\pi(e) \in[m, M] \quad \text { and } \quad \widehat{\Delta}^{\widehat{u}, e}(t, \widehat{x}, y, \psi) \geq \eta \quad \text { for } \lambda \text {-a.e. } e \in E\right\} \\
\widehat{H}_{\varepsilon, \eta}(\widehat{\Theta}, \varphi):=\underset{\widehat{u} \in \widehat{\mathcal{N}}_{\varepsilon, \eta}(t, \widehat{x}, y, q, \varphi)}{\sup ^{(} \widehat{\mathbf{A}}^{\widehat{u}}(\widehat{\Theta})}
\end{gathered}
$$

where

$$
\begin{gathered}
\widehat{\Theta}:=(t, \widehat{x}, y, k, q, A) \\
\widehat{\mathbf{A}}^{\widehat{u}}(\widehat{\Theta}):=-k+\mu_{Y}(x, y, u)-\widehat{\mu}(\widehat{x}, \widehat{u}) \cdot q-\frac{1}{2} \operatorname{Tr}\left[\widehat{\sigma} \widehat{\sigma}^{T}(\widehat{x}, \widehat{u}) A\right]
\end{gathered}
$$

and

$$
\begin{gathered}
\widehat{\mathbf{N}}(t, \widehat{x}, y, q, \psi):=\left\{(r, s) \in \mathbb{R}^{d} \times \mathbb{R}: \exists \widehat{u} \in \widehat{U} \text { s.t. } r=\widehat{N}^{\widehat{u}}(\widehat{x}, y, q)\right. \\
\text { and } \left.s \leq \widehat{\Delta}^{\widehat{u}, e}(t, \widehat{x}, y, \psi) \quad \text { for } \lambda \text {-a.e. } e \in E\right\}, \\
\widehat{\delta}:=\operatorname{dist}\left(0, \widehat{\mathbf{N}}^{c}\right)-\operatorname{dist}(0, \widehat{\mathbf{N}}) .
\end{gathered}
$$

The operators $\widehat{H}^{*}, \widehat{H}_{*} \widehat{\delta}^{*}$ and $\widehat{\delta}_{*}$ are constructed from $\widehat{H}_{\varepsilon, \eta}$ and $\widehat{\delta}$ exactly as $H^{*}, H_{*}, \delta^{*}$ and $\delta_{*}$ are defined from $H_{\varepsilon, \eta}$ and $\delta$. Finally, we define the function

$$
\widehat{g}(\widehat{x}):=\inf \{y \geq-\kappa: \widehat{V}(\widehat{x}, y) \geq 0\}, \quad \widehat{x}=(x, p) \in \mathbf{X} \times[m, M] .
$$

As an almost direct consequence of Theorem 2.1 and (3.2), we obtain the viscosity property of $\widehat{v}$ under the following assumption, which is the analog of Assumption 2.1 for the augmented control system $\widehat{\mathbf{X}}$ :

Assumption 3.2. (Continuity of $\left.\widehat{\mathcal{N}}_{0, \eta}(t, x, p, y, q, f)\right)$ Let $B$ be a subset of $[0, T] \times \boldsymbol{X} \times[m, M] \times$ $\mathbb{R} \times \mathbb{R}^{d+1}, f \in \mathcal{C}^{0}([0, T] \times \boldsymbol{X} \times[m, M])$ and $\eta>0$ such that $\widehat{\mathcal{N}}_{0, \eta}(\cdot, f) \neq \emptyset$ on $B$. Then, for every $\varepsilon>0,\left(t_{0}, x_{0}, p_{0}, y_{0}, q_{0}\right) \in \operatorname{Int}(B)$ and $\widehat{u}_{0} \in \widehat{\mathcal{N}}_{0, \eta}\left(t_{0}, x_{0}, p_{0}, y_{0}, q_{0}, f\right)$, there exists an open neighborhood $B^{\prime}$ of $\left(t_{0}, x_{0}, p_{0}, y_{0}, q_{0}\right)$ and a locally Lipschitz map $\widehat{\nu}$ defined on $B^{\prime}$ such that $\left|\widehat{\nu}\left(t_{0}, x_{0}, p_{0}, y_{0}, q_{0}\right)-\widehat{u}_{0}\right| \leq \varepsilon$, and $\widehat{\nu}(t, x, p, y, q) \in \widehat{\mathcal{N}}_{0, \eta}(t, x, y, p, q, f)$ on $B^{\prime}$.

Corollary 3.1. The function $\widehat{v}_{*}$ is a viscosity supersolution of

$$
\widehat{H}^{*} \widehat{v}_{*} \geq \text { on }[0, T) \times \widehat{\boldsymbol{X}} .
$$

Under the additional Assumption 3.2, $\widehat{v}^{*}$ is a viscosity subsolution of

$$
\min \left\{\widehat{v}^{*}+\kappa, \widehat{H}_{*} \widehat{v}^{*}\right\} \leq 0 \text { on }[0, T) \times \widehat{\boldsymbol{X}}
$$


The supersolution property is a direct consequence of Theorem 2.1, the representation (3.2) and 3. of Remark 3.1. The subsolution property is obtained similarly.

Example 3.3. In the context of both Example 3.1 and Example 3.2, with the dynamics of Example 2.1, the conditions of Theorem 3.1 are trivially satisfied. By direct computations, we then have that both $\check{v}_{*}$ and $\widetilde{v}_{*}$ are viscosity supersolution on $[0, T) \times \mathbf{X}$ of

$$
\begin{aligned}
0 \leq-\partial_{t} \varphi & -\frac{1}{2} \sigma^{2} D_{x x} \varphi \\
- & \inf _{\substack{\pi \in \Pi(p) \\
\alpha \in \mathbb{R}^{d}}}\left\{\frac{1}{2}|\alpha|^{2} D_{p p} \varphi+\operatorname{Tr}\left[\sigma \alpha D_{x p} \varphi\right]-\alpha\left(D_{p} \varphi\right)^{T} \sigma^{-1} \mu-D_{p} \varphi \int_{E} \pi(e) \lambda(d e)\right\},
\end{aligned}
$$

whenever $D_{p p} \varphi>0$, and with

$$
\begin{array}{r}
\Pi(p):=\left\{\pi \in \mathbb{L}_{\lambda}^{2} \text { s.t. }\left(D_{x} \varphi+\sigma^{-1} D_{p} \varphi \alpha\right) \beta(\cdot, e)-\varphi(\cdot, \cdot+\beta(\cdot, e), \cdot+\pi(e))+\varphi \geq 0\right. \\
\text { and } p+\pi \in[0,1] \text { for } \lambda \text {-a.e. } e \in E\} .
\end{array}
$$

\subsection{Boundary conditions and state constraint}

In our general context, the natural domain of $P$ is $[m, M]$. In the case where $m$ or $M$ are finite, we need to specify the boundary conditions at the end points $m$ and $M$.

By definition of the stochastic target problem with controlled expected loss, we have

$$
\widehat{v}(\cdot, M)=v \text { and } \widehat{v}(\cdot, m)=-\kappa,
$$

where

$$
v(t, x):=\inf \left\{y \geq-\kappa: \Phi\left(X_{t, x}^{\nu}(T), Y_{t, x, y}^{\nu}(T)\right) \geq 0 \text { for some } \nu \in \mathcal{U}\right\}
$$

with

$$
\Phi(x, y):=\Psi(x, y)-M .
$$

Also, since $\Psi$ is non-decreasing in $y$, we know that $\widehat{v}$ is non-decreasing in $p$. Hence,

$$
\begin{gathered}
-\kappa \leq \widehat{v}_{*}(\cdot, m) \leq \widehat{v}^{*}(\cdot, p) \leq \widehat{v}^{*}(\cdot, M) \leq v^{*} \text { for } p \in[m, M], \\
\widehat{v}^{*}(\cdot, p)=-\kappa \text { for } p<m \text { and } \widehat{v}^{*}(\cdot, p)=\infty \text { for } p>M,
\end{gathered}
$$

and one can naturally expect that $\widehat{v}_{*}(\cdot, m)=-\kappa$ and $\widehat{v}^{*}(\cdot, M)=v^{*}$. However, the function $\widehat{v}$ may have discontinuities at $p=m$ or $p=M$ and, in general, the boundary conditions have to be stated in a weak form, see (3.17) and (3.52) below. This corresponds to the classical state-space constraint problems, see [1], [5], [7] or [8] and the references therein.

To obtain a characterization of $\widehat{v}$ on these boundaries, we shall appeal to the following additional assumptions. Assumption 3.5 and Assumption 3.6 already appeared in [3]. Assumption 3.3, Assumption 3.4 and Assumption 3.7 will be used to handled the non-local operator.

Assumption 3.3. H1: For some integer $\gamma \geq 1, \widehat{v}^{*}(\cdot, m)^{+}$satisfies the growth condition

$$
\sup _{[0, T] \times \mathbb{R}^{d}} \frac{|w(t, x)|}{1+|x|^{\gamma}}<\infty .
$$

H2: There is a function $\Lambda$ on $\mathbb{R}^{d}$ satisfying

(i) For all $x \in \boldsymbol{X}$ and $y>\Lambda(x)$, there exists $\bar{u} \in U$ such that

$$
\beta_{Y}(x, y, \bar{u}(e), e)-\Lambda\left(x+\beta_{X}(x, \bar{u}(e), e)\right)+\Lambda(x)>0 \quad \text { for } \lambda \text {-a.e. } e \in E \text {. }
$$

(ii) $\Lambda(x) /|x|^{\gamma} \rightarrow+\infty$ as $|x| \rightarrow \infty$. 
(iii) $\Lambda \leq-\kappa$ on $\boldsymbol{X}$.

Assumption 3.4. The set $E$ is finite and $\lambda(e)>0$ for all $e \in E$.

Assumption 3.5. For all $(x, y, q) \in \boldsymbol{X} \times(-\kappa, \infty) \times \mathbb{R}^{d}$, we have $\left\{u \in U: N^{u}(x, y, q)=0\right\} \varsubsetneqq U$.

We need for the next assumption to introduce the following set, for $(x, y, q) \in \mathbb{R}^{d} \times \mathbb{R} \times \mathbb{R}^{d}$ :

$$
\widetilde{\mathcal{N}}_{\varepsilon}(x, y, q):=\left\{u \in U:\left|N^{u}(x, y, q)\right| \leq \varepsilon\right\} .
$$

Assumption 3.6. For all compact subset $D$ of $\mathbb{R}^{d} \times \mathbb{R} \times \mathbb{R} \times \mathbb{R}^{d} \times \mathbb{S}^{d}$, there exists $C>0$ such that

$$
\sup _{u \in \widetilde{\mathcal{N}}_{\varepsilon}(x, y, q)}\left\{\mu_{Y}(x, y, u)-k-\mu_{X}(x, u) \cdot q-\frac{1}{2} \operatorname{Tr}\left[\sigma_{X} \sigma_{X}^{T}(x, u) A\right]\right\} \leq C\left(1+\varepsilon^{2}\right)
$$

for all $\varepsilon>0$ and $(x, y, k, q, A) \in D$.

Assumption 3.7. The maps $\beta_{X}, \beta_{Y}$ are continuous on $\boldsymbol{X} \times E$ and $\boldsymbol{X} \times \mathbb{R} \times E$ uniformly in $u \in U$. Moreover, $\beta_{X}, \beta_{Y}$ and $\sigma_{X}$ satisfy the following condition

$$
\text { ess } \sup _{u \in U, e \in E}\left\{\left|\beta_{X}(\cdot, u(e), e)\right|+\left|\beta_{Y}(\cdot, u(e), e)\right|+\left|\sigma_{X}(\cdot, u)\right|\right\} \text { is locally bounded }
$$

Since the main concern of this paper is the analysis of the stochastic target problem under controlled loss with jumps, we do not establish a comparison result of viscosity supersolutions of (2.6)-(2.9) and subsolutions of (2.7)-(2.10). Nonetheless, as in [3], we need such a comparison result in order to establish the boundary conditions of this section.

Assumption 3.8. There is a class of functions $\mathcal{C}$ containing all $[-\kappa,+\infty)$ valued functions dominated by $v^{*}$ such that, for every

- $v_{1} \in \mathcal{C}$, lower semi-continuous viscosity supersolution of (2.6)-(2.9) on $[0, T] \times \boldsymbol{X}$

- $v_{2} \in \mathcal{C}$, upper semi-continuous viscosity subsolution of (2.7)-(2.10) on $[0, T] \times \boldsymbol{X}$

we have $v_{1} \geq v_{2}$.

The main results of this section shows that the natural boundary conditions (3.10) indeed holds true, whenever the comparison principle of Assumption 3.8 holds and under the above additional conditions.

Theorem 3.1. Assume that Assumption 3.2, Assumption 3.4 and Assumption 3.7 hold true.

(i) Assume that $m>-\infty$. Under Assumption 3.3, and Assumption 3.5, we have $\widehat{v}^{*}(\cdot, m)=-\kappa$ on $[0, T) \times \boldsymbol{X}$ and $\widehat{v}_{*}(\cdot, m)=-\kappa$ on $[0, T] \times \boldsymbol{X}$.

(ii) Assume that $M<\infty$. Under Assumption 3.6, $\widehat{v}^{*}(\cdot, M)$ is a viscosity supersolution of (2.6)(2.9) on $[0, T] \times \boldsymbol{X}$. In particular, if in addition the comparison principle of Assumption 3.8 is satisfied, then $\widehat{v}^{*}(\cdot, M)=\widehat{v}_{*}(\cdot, M)=v_{*}=v^{*}$ on $[0, T] \times \boldsymbol{X}$.

The proof is reported in Section 3.5.

Remark 3.2. This subsection is similar to the one in [3], where the authors studied the boundary conditions at $p=0$ and $p=1$ in the case of target reachability under controlled probability, i.e. $\Psi$ is of the form $\Psi(x, y)=\mathbb{1}_{\{y \geq g(x)\}}$. In this paper, the natural domain of $P$ is $[0,1]$, and the authors studied the behavior of the value function $\widehat{v}$ when $p \rightarrow 0$ and $p \rightarrow 1$.

\subsection{On the Terminal Condition}

The boundary condition at $T$ for $\widehat{v}_{*}$ and $\widehat{v}^{*}$ can be easily derived from the characterization of Theorem 2.2.

Corollary 3.2. The function $\widehat{x} \in \widehat{\boldsymbol{X}} \mapsto \widehat{v}_{*}(T, \widehat{x})$ is a viscosity supersolution of

$$
\min \left\{\left(\widehat{v}_{*}(T, \cdot)-\widehat{g}_{*}\right) \mathbb{1}_{\left\{\widehat{H}^{*} \widehat{v}_{*}(T, \cdot)<\infty\right\}}, \widehat{\delta}^{*} \widehat{v}_{*}(T, \cdot)\right\} \geq 0 \text { on } \widehat{\boldsymbol{X}}
$$

If in addition, Assumption 3.2 holds, then $\widehat{x} \in \widehat{\boldsymbol{X}} \mapsto \widehat{v}^{*}(T, \widehat{x})$ is a viscosity subsolution of

$$
\min \left\{\widehat{v}^{*}(T, \cdot)-\widehat{g}^{*}, \widehat{\delta}_{*} \widehat{v}^{*}(T, \cdot)\right\} \leq 0 \text { on } \widehat{\boldsymbol{X}}
$$


The condition $\widehat{H}^{*} \widehat{v}_{*}(T, \cdot)<\infty$ may not be satisfied because the control $(\alpha, \chi)$ appearing in the definition of $\widehat{H}$ may not be bounded. It follows that the above boundary condition may be useless in most examples.

The rest of this section is devoted to the discussion of conditions under which a precise boundary condition can be specified.

Proposition 3.2. (i) Assume that for all sequence $\left(t_{n}, x_{n}, y_{n}, p_{n}, \nu_{n}\right)_{n \geq 1}$ of $[0, T) \times \boldsymbol{X} \times \mathbb{R}_{+} \times$ $[m, M] \times \mathcal{U}$ such that $\left(t_{n}, x_{n}, y_{n}, p_{n}\right) \rightarrow(T, x, y, p) \in\{T\} \times \boldsymbol{X} \times \mathbb{R}_{+} \times[m, M]$, there exists a sequence of $\mathbb{P}$-absolutely continuous probability measure $\left(\mathbb{Q}^{n}\right)_{n>1}$, defined by $\frac{d \mathbb{Q}^{n}}{d \mathbb{P}}=: H^{n}$ for some sequence of non-negative random variable $\left(H^{n}\right)_{n \geq 1}$, such that

$$
\begin{gathered}
\limsup _{n \rightarrow \infty} \mathbb{E}^{\mathbb{Q}^{n}}\left[Y_{t_{n}, x_{n}, y_{n}}^{\nu_{n}}\right] \leq y, \quad \limsup _{n \rightarrow \infty} \mathbb{E}\left[\left|H^{n} D_{p}^{+} \odot \widehat{g}\left(X_{t_{n}, x_{n}}^{\nu_{n}}(T), p_{n}\right)-D_{p}^{+} \odot \widehat{g}\left(x_{n}, p_{n}\right)\right|\right]=0 \\
\text { and } \quad \liminf _{n \rightarrow \infty} \mathbb{E}\left[H^{n} \odot \widehat{g}\left(X_{t_{n}, x_{n}}^{\nu_{n}}(T), p_{n}\right)\right] \geq \odot \widehat{g}(x, p),
\end{gathered}
$$

where $D_{p}^{+}$stands for the right derivative in $p$.

Then, $\widehat{v}_{*}(T, x, p) \geq \odot \widehat{g}(x, p)$ for all $(x, p) \in \boldsymbol{X} \times[0,1]$.

(ii) Let the conditions (ii) of Theorem 3.1 hold true and assume that $\widehat{v}^{*}$ is convex in its p-variable and that $v^{*}(T, x) \leq g(x)$. Then $\widehat{v}^{*}(T, x, p) \leq \odot \widehat{g}(x, p)$ for all $(x, p) \in \boldsymbol{X} \times[m, M]$.

Proof. (i) Given a sequence $\left(t_{n}, x_{n}, p_{n}\right)_{n \geq 1}$ in $[0, T) \times \mathbf{X} \times(m, M)$ such that $\left(t_{n}, x_{n}, p_{n}\right) \rightarrow$ $(T, x, p)$ and $\widehat{v}\left(t_{n}, x_{n}, p_{n}\right) \rightarrow \widehat{v}_{*}(T, x, p)$, we can find $\widehat{\nu}_{n}=\left(\nu_{n}, \alpha_{n}, \chi_{n}\right) \in \widehat{\mathcal{U}}$ such that

$$
\widehat{V}\left(\widehat{X}_{t_{n}, x_{n}, p_{n}}^{\widehat{\nu}_{n}}(T), Y_{t_{n}, x_{n}, y_{n}}^{\widehat{\nu}_{n}}(T)\right) \geq 0,
$$

where $y_{n}:=\widehat{v}\left(t_{n}, x_{n}, p_{n}\right)+n^{-1} \rightarrow \widehat{v}_{*}(T, x, p)$, recall (3.2). This implies that

$$
Y_{t_{n}, x_{n}, y_{n}}^{\widehat{\nu}_{n}}(T) \geq \widehat{g}\left(\widehat{X}_{t_{n}, x_{n}, p_{n}}^{\widehat{\nu}_{n}}(T)\right)
$$

and, by the definition of the convex hull of $\widehat{g}$,

$$
H^{n} Y_{t_{n}, x_{n}, y_{n}}^{\widehat{\nu}_{n}}(T) \geq H^{n} \odot \widehat{g}\left(\widehat{X}_{t_{n}, x_{n}, p_{n}}^{\widehat{\nu}_{n}}(T)\right) .
$$

Using the convexity of $\odot \widehat{g}$ then leads to

$$
\begin{aligned}
H^{n} Y_{t_{n}, x_{n}, y_{n}}^{\widehat{\nu}_{n}}(T) \geq & H^{n} \odot \widehat{g}\left(X_{t_{n}, x_{n}}^{\nu_{n}}(T), p_{n}\right)+H^{n} D_{p}^{+} \odot \widehat{g}\left(X_{t_{n}, x_{n}}^{\nu_{n}}(T), p_{n}\right)\left(P_{t_{n}, p_{n}}^{\alpha_{n}, \chi_{n}}(T)-p_{n}\right) \\
= & H^{n} \odot \widehat{g}\left(X_{t_{n}, x_{n}}^{\nu_{n}}(T), p_{n}\right)+D_{p}^{+} \odot \widehat{g}\left(x_{n}, p_{n}\right) P_{t_{n}, p_{n}}^{\alpha_{n}, \chi_{n}}(T) \\
& -H^{n} p_{n} D_{p}^{+} \odot \widehat{g}\left(X_{t_{n}, x_{n}}^{\nu_{n}}(T), p_{n}\right) \\
& +P_{t_{n}, p_{n}}^{\alpha_{n}, \chi_{n}}(T)\left[H^{n} D_{p}^{+} \odot \widehat{g}\left(X_{t_{n}, x_{n}}^{\nu_{n}}(T), p_{n}\right)-D_{p}^{+} \odot \widehat{g}\left(x_{n}, p_{n}\right)\right] \\
\geq & H^{n} \odot \widehat{g}\left(X_{t_{n}, x_{n}}^{\nu_{n}}(T), p_{n}\right)+D_{p}^{+} \odot \widehat{g}\left(x_{n}, p_{n}\right) P_{t_{n}, p_{n}}^{\alpha_{n}, \chi_{n}}(T) \\
& -H^{n} p_{n} D_{p}^{+} \odot \widehat{g}\left(X_{t_{n}, x_{n}}^{\nu_{n}}(T), p_{n}\right) \\
& -M\left|H^{n} D_{p}^{+} \odot \widehat{g}\left(X_{t_{n}, x_{n}}^{\nu_{n}}(T), p_{n}\right)-D_{p}^{+} \odot \widehat{g}\left(x_{n}, p_{n}\right)\right|
\end{aligned}
$$

where the last inequality follows from the fact that we can always assume that $P_{t_{n}, p_{n}}^{\alpha_{n}, \chi_{n}}$ takes values in $[m, M]$, see (3.3). Taking the expectation under $\mathbb{P}$ and using the fact that $P_{t_{n}, p_{n}}^{\alpha_{n}, \chi_{n}}$ is a $\mathbb{P}$-martingale, we obtain

$$
\begin{aligned}
& \mathbb{E}^{\mathbb{Q}^{n}} {\left[Y_{t_{n}, x_{n}, y_{n}}^{\widehat{\nu}_{n}}(T)\right] } \\
& \geq \mathbb{E}\left[H^{n} \odot \widehat{g}\left(X_{t_{n}, x_{n}}^{\nu_{n}}(T), p_{n}\right)+p_{n}\left(D_{p}^{+} \odot \widehat{g}\left(x_{n}, p_{n}\right)-H^{n} D_{p}^{+} \odot \widehat{g}\left(X_{t_{n}, x_{n}}^{\nu_{n}}(T), p_{n}\right)\right)\right. \\
&\left.\quad-M\left|H^{n} D_{p}^{+} \odot \widehat{g}\left(X_{t_{n}, x_{n}}^{\nu_{n}}(T), p_{n}\right)-D_{p}^{+} \odot \widehat{g}\left(x_{n}, p_{n}\right)\right|\right] .
\end{aligned}
$$

Passing to the limit, and using (3.15) leads to $\widehat{v}_{*}(T, x, p) \geq \odot \widehat{g}(x, p)$.

(ii) Using (3.12) and the convexity of $\widehat{v}^{*}$ together with the definition of the convex hull of a function lead to the required result. 
Example 3.4. In the context of Example 3.1, we may easily notice that the generalized inverse of $\check{V}$ at 0 ,

$$
\check{g}(x, p):=\inf \{y \geq-\kappa: \check{V}(x, p, y) \geq 0\},
$$

satisfies

$$
\check{g}(x, p)=p g(x)
$$

and is convex in $p$. Moreover, for the dynamics of Example 2.1, the convexity of $\check{v}$ in its $p$ variable is quite obvious, since $Y_{t, x, \mu y}^{\nu}(T)=\mu Y_{t, x, y}^{\nu}(T)$ for any $\mu \in[0,1]$, and the expectation operator is linear.

We have already shown in Section 3.2 that $\check{v}_{*}$ is a supersolution of (3.9). Notice that the condition of Corollary 3.1 and (i) of Proposition 3.2 are satisfied. In this case, we deduce that $\check{v}_{*}$ satisfies the boundary conditions

$$
\check{v}_{*}(\cdot, 1)=v \text { and } \check{v}_{*}(\cdot, 0)=0 \text { on }[0, T) \times \mathbf{X} \text { and } \check{v}_{*}(T, x, p) \geq p g(x) \text { on } \mathbf{X} \times[0,1] .
$$

Example 3.5. In the context of Example 3.2, we define the function

$$
\widetilde{g}(x, p):=\inf \{y \geq-\kappa: \widetilde{V}(x, p, y) \geq 0\}
$$

and let $\widetilde{\psi}$ be the generalized inverse of $\widetilde{\Psi}$ at 0 , i.e. $\widetilde{\psi}(x):=\inf \{y \geq-\kappa: \widetilde{\Psi}(x, y) \geq 0\}$. Then, $\widetilde{g}(x, p)=\widetilde{\psi}(x) \mathbb{1}_{\{p>0\}}$ for $x \in \mathbf{X}$ and $p \in[0,1]$. The convexity of $\widetilde{v}$ is far from being obvious. However, one may notice that the convex hull of $\widetilde{g}$ in $p$ is $\odot(\widehat{g})(x, p)=p g(x)$, with $g=\widetilde{\psi}^{-1}$, and that the condition of Corollary 3.1 and (i) of Proposition 3.2 are satisfied. It follows that, as for the expected success ratio problem of Example 3.4 above, $\widetilde{v}_{*}$ is a viscosity supersolution on $[0, T] \times \mathbf{X} \times[0,1]$ of $(3.9)-(3.16)$.

Remark 3.3. In [3], the authors considered the case $\widehat{g}(x, p)=g(x) \mathbb{1}_{\{p>0\}}$, so that $\odot \widehat{g}(x, p)=$ $p g(x)$, and therefore $D_{p}^{+} \odot \widehat{g}(x, p)=g(x)$. Then, Assumption (3.15), in the case of [3], should take the form:

$$
\limsup _{n \rightarrow \infty} \mathbb{E}\left[\left|H^{n} g\left(X_{t_{n}, x_{n}}^{\nu_{n}}(T)\right)-g(x)\right|\right]=0 .
$$

The Assumption (3.15) is then almost the counterpart of the one made in their proposition 3.2. The difference comes from a slight error in their $\operatorname{proof}^{2}$ where they use the fact that $P_{t_{n}, p_{n}}^{\alpha_{n}} \chi_{n}$ is a $\mathbb{Q}$-martingale while it is only a $\mathbb{P}$-martingale, a priori.

\subsection{Derivation of the boundary conditions for the stochastic target with controlled expected loss}

We now prove Theorem 3.1. These boundary conditions need only to be specified in the case where $m$ and/or $M$ are finite.

In all this section, we shall use the following notations

$$
\begin{aligned}
\widehat{\mathcal{L}}^{\widehat{u}} \varphi(t, \widehat{x}) & :=\partial_{t} \varphi(t, \widehat{x})+\widehat{\mu}(\widehat{x}, \widehat{u}) \cdot D \varphi(t, \widehat{x})+\frac{1}{2} \operatorname{Tr}\left[\widehat{\sigma} \widehat{\sigma}^{T}(\widehat{x}, \widehat{u}) D^{2} \varphi(t, \widehat{x})\right] \\
\widehat{\mathcal{G}}^{\widehat{u}, e} \varphi(t, \widehat{x}) & :=\beta_{Y}(x, \varphi(t, \widehat{x}), u(e), e)-\varphi(t, \widehat{x}+\widehat{\beta}(\widehat{x}, \widehat{u}(e), e))+\varphi(t, \widehat{x}),
\end{aligned}
$$

for $\widehat{x}=(x, p), \widehat{u}=(u, \alpha, \chi)$.

\footnotetext{
${ }^{2}$ The author would like to thank Bruno Bouchard, Romuald Elie and Nizar Touzi for pointing out this issue and for their explanations on how to fix it in their particular context.
} 


\subsubsection{The endpoint $p=M$, finite}

In order to show that $\widehat{v}_{*}(\cdot, M)$ is a viscosity supersolution of (2.6)-(2.9), it suffices to show that $\widehat{v}_{*}(\cdot, M)$ is a viscosity supersolution on $[0, T) \times \mathbf{X}$ of

$$
\max \left\{\widehat{v}_{*}(\cdot, M)-v_{*}, H^{*} \widehat{v}_{*}(\cdot, M)\right\} \geq 0,
$$

and that $\widehat{v}_{*}(T, \cdot, M)$ is a viscosity supersolution on $\mathbf{X}$ of

$$
\max \left\{\widehat{v}_{*}(T, \cdot, M)-v_{*}, \min \left\{\left(\widehat{v}_{*}(T, \cdot, M)-j_{*}\right) \mathbb{1}_{\left\{H_{*} \widehat{v}_{*}(T, \cdot, M)<\infty\right\}}, \delta^{*} \widehat{v}_{*}(T, \cdot, M)\right\}\right\} \geq 0,
$$

where $j$ is the generalized inverse of $\Phi$ at 0 :

$$
j(x):=\inf \{y \geq-\kappa: \Phi(x, y) \geq 0\}
$$

recall $(3.11)$.

To convince ourself, let us show for instance that (3.17) implies (2.6). Let $\left(t_{0}, x_{0}\right)$ be a local minimizer of $\widehat{v}_{*}(\cdot, M)-\varphi$ for some smooth function $\varphi$. Then

- either $\widehat{v}_{*}\left(t_{0}, x_{0}, M\right)<v_{*}\left(t_{0}, x_{0}\right)$ and then (2.6) holds for $\varphi$ at $\left(t_{0}, x_{0}\right)$

- or $\widehat{v}_{*}\left(t_{0}, x_{0}, M\right)=v_{*}\left(t_{0}, x_{0}\right)$ so that $\left(t_{0}, x_{0}\right)$ is a local minimizer of $v_{*}-\varphi$, and (2.6) holds for $\varphi$ at $\left(t_{0}, x_{0}\right)$ by the viscosity property of $v_{*}$, see Theorem 2.1 .

$1^{\text {st }}$ step: We first show that for any smooth function $\varphi$ on $[0, T] \times \mathbf{X} \times[m, M]$ and $\left(t_{0}, x_{0}\right) \in$

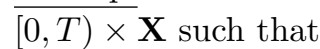

$$
\text { (strict) } \min _{[0, T) \times \mathbf{X} \times[m, M]}\left(\widehat{v}_{*}-\varphi\right)=\left(\widehat{v}_{*}-\varphi\right)\left(t_{0}, x_{0}, M\right)=0,
$$

we have

$$
\max \left\{\varphi\left(t_{0}, x_{0}, M\right)-v_{*}\left(t_{0}, x_{0}\right), \widehat{H}^{*} \varphi\left(t_{0}, x_{0}, M\right)\right\} \geq 0 .
$$

If not, we can find $\eta, \varepsilon, \iota>0$ such that

$$
\begin{gathered}
\max \left\{\varphi_{\iota}-v_{*}(t, x), \mu_{Y}(x, y, u)-\widehat{\mathcal{L}}^{\widehat{u}} \varphi_{\iota}(t, x, p),\right\} \leq-\eta \\
\text { for all } \widehat{u}:=(u, \alpha, \pi) \in \widehat{\mathcal{N}}_{\varepsilon,-\eta}\left(t, x, y, D \varphi_{\iota}(t, x, p), \varphi_{\iota}\right) \\
\text { and }(t, x, p, y) \in[0, T) \times \mathbf{X} \times(m, M] \times \mathbb{R}
\end{gathered}
$$

s.t. $(t, x, p) \in B_{\varepsilon}\left(t_{0}, x_{0}\right) \times[M-\varepsilon, M] \quad$ and $\quad\left|y-\varphi_{\iota}(t, x, p)\right| \leq \varepsilon$,

with $\varphi_{\iota}(t, x, p):=\varphi(t, x, p)-\iota\left(\left|x-x_{0}\right|^{4}+|p-M|^{4}\right)$.

Let $\left(t_{n}, x_{n}, p_{n}\right)_{n}$ be a sequence in $[0, T) \times \mathbf{X} \times(m, M)$ which converges to $\left(t_{0}, x_{0}, M\right)$ and such that $\widehat{v}\left(t_{n}, x_{n}, p_{n}\right) \rightarrow \widehat{v}_{*}\left(t_{0}, x_{0}, M\right)$. Set $y_{n}:=\widehat{v}\left(t_{n}, x_{n}, p_{n}\right)+n^{-1}$ and observe that

$$
\gamma_{n}:=y_{n}-\varphi_{\iota}\left(t_{n}, x_{n}, p_{n}\right) \rightarrow 0 .
$$

For each $n \geq 1$, we have $y_{n}>\widehat{v}\left(t_{n}, x_{n}, p_{n}\right)$. Then, by (GDP1), there exists some $\widehat{\nu}^{n}:=$ $\left(\nu^{n}, \alpha^{n}, \chi^{n}\right) \in \widehat{\mathcal{U}}$ such that

$$
Y^{n}\left(\theta_{n}\right) \geq \widehat{v}_{*}\left(\theta_{n}, X^{n}\left(\theta_{n}\right), P^{n}\left(\theta_{n}\right)\right) \geq \varphi_{\iota}\left(\theta_{n}, X^{n}\left(\theta_{n}\right), P^{n}\left(\theta_{n}\right)\right)
$$

where

$$
\begin{aligned}
& \theta_{n}^{o}:=\left\{s \geq t_{n}:\left(s, X^{n}(s), P^{n}(s)\right) \in D\right\} \\
& \theta_{n}:=\left\{s \geq t_{n}:\left|Y^{n}(s)-\varphi_{\iota}\left(s, X^{n}(s), P^{n}(s)\right)\right| \geq \varepsilon\right\} \wedge \theta_{n}^{o}
\end{aligned}
$$

together with

$$
\widehat{Z}^{n}:=\left(X^{n}, P^{n}, Y^{n}\right):=\left(X_{t_{n}, x_{n}}^{\nu^{n}}, P_{t_{n}, p_{n}}^{\alpha^{n}, \chi^{n}}, Y_{t_{n}, x_{n}, y_{n}}^{\nu^{n}}\right)
$$


and

$$
\begin{aligned}
& \mathcal{V}_{\varepsilon}\left(t_{0}, x_{0}\right):=\left(\left\{t_{0}+\varepsilon\right\} \times B_{\varepsilon}\left(x_{0}\right)\right) \cup\left(\left[t_{0}, t_{0}+\varepsilon\right) \times B_{\varepsilon}^{c}\left(x_{0}\right)\right) \\
& D:=\left(\mathcal{V}_{\varepsilon}\left(t_{0}, x_{0}\right) \times[M-\varepsilon, M]\right) \cup\left(B_{\varepsilon}\left(t_{0}, x_{0}\right) \times[m, M-\varepsilon]\right) .
\end{aligned}
$$

It follows from (3.20) and (3.19)

$$
\zeta:=\inf _{D}\left(\widehat{v}-\varphi_{\iota}\right)>0 .
$$

Using the definition of $\theta_{n}$ and $\zeta>0$, this implies that

$$
Y^{n}\left(\theta_{n}\right)-\varphi_{\iota}\left(\theta_{n}, X^{n}\left(\theta_{n}\right), P^{n}\left(\theta_{n}\right)\right) \geq \zeta \wedge \varepsilon .
$$

By arguing as in Section 2.3.1, this leads to a contradiction.

$2^{\text {nd }}$ step: We now show (3.17), i.e. for any smooth function $\varphi$ on $[0, T] \times \mathbf{X}$ and $\left(t_{0}, x_{0}\right) \in$ $[0, T) \times \mathbf{X}$ such that

$$
\text { (strict) } \min _{[0, T) \times \mathbf{X}}\left(\widehat{v}_{*}(\cdot, M)-\varphi\right)=\left(\widehat{v}_{*}(\cdot, M)-\varphi\right)\left(t_{0}, x_{0}\right)=0,
$$

we have

$$
\max \left\{\varphi\left(t_{0}, x_{0}\right)-v_{*}\left(t_{0}, x_{0}\right), H^{*} \varphi\left(t_{0}, x_{0}\right)\right\} \geq 0 .
$$

a. The first step is similar as in [3]. For every $k$, we introduce the smooth function

$$
\varphi_{k}(t, x, p):=\varphi(t, x)-\left(\left|x-x_{0}\right|^{4}+\left(t-t_{0}\right)^{2}+\psi_{k}(p)\right),
$$

where, for some $\rho>0$,

$$
\psi_{k}(p):=-\rho k \int_{p}^{M} \frac{e^{2 k M}}{e^{k(r+M)}-e^{2 k M+1}} d r, \quad k>0 .
$$

Observe that

$$
\psi_{k}(p) \geq 0 \text { for all } k>0, p \in[m, M],
$$

$$
\begin{gathered}
-2 \rho k \leq \psi_{k}^{\prime}(p)=\rho k \frac{e^{2 k M}}{e^{k(p+M)}-e^{2 k M+1}} \leq-\frac{\rho k}{2(e-1)} \quad \text { for } k \text { large enough, } \\
\psi_{k}^{\prime \prime}(p)=-\rho k^{2} \frac{e^{k(p+3 M)}}{\left(e^{k(p+M)}-e^{2 k M+1}\right)^{2}}<0 \quad \text { for all } k>0,
\end{gathered}
$$

$$
\lim _{k \rightarrow \infty} \frac{\left(\psi_{k}^{\prime}\left(p_{k}\right)\right)^{2}}{\left|\psi_{k}^{\prime \prime}\left(p_{k}\right)\right|}=\rho \quad \text { if }\left(p_{k}\right)_{k} \text { is a sequence in }[m, M] \text { s.t. } \quad \lim _{k \rightarrow \infty} k\left(M-p_{k}\right)=0 \text {. }
$$

Let $\left(t_{k}, x_{k}, p_{k}\right)$ be a minimizer of $\widehat{v}_{*}-\varphi_{k}$ on $[0, T] \times \overline{B_{1}^{\mathbf{X}}\left(x_{0}\right)} \times[m, M]$, where $B_{1}^{\mathbf{X}}\left(x_{0}\right):=B_{1}\left(x_{0}\right) \cap \mathbf{X}$ and $B_{1}\left(x_{0}\right)$ is the open unit ball centered at $x_{0}$. Observe that, by definition of $\left(t_{k}, x_{k}, p_{k}\right)$ and $\left(t_{0}, x_{0}\right)$,

$$
\begin{aligned}
\left(\widehat{v}_{*}(\cdot, M)-\varphi\right)\left(t_{0}, x_{0}\right) & =\left(\widehat{v}_{*}-\varphi_{k}\right)\left(t_{0}, x_{0}, M\right) \\
& \geq\left(\widehat{v}_{*}-\varphi_{k}\right)\left(t_{k}, x_{k}, p_{k}\right) \\
& =\left(\widehat{v}_{*}\left(\cdot, p_{k}\right)-\varphi\right)\left(t_{k}, x_{k}\right)+\left(\left|x_{k}-x_{0}\right|^{4}+\left(t_{k}-t_{0}\right)^{2}+\psi_{k}\left(p_{k}\right)\right) \\
& \geq\left(\widehat{v}_{*}\left(\cdot, p_{k}\right)-\varphi\right)\left(t_{k}, x_{k}\right)+\left(\left|x_{k}-x_{0}\right|^{4}+\left(t_{k}-t_{0}\right)^{2}+\frac{\rho k}{2(e-1)}\left(M-p_{k}\right)\right),
\end{aligned}
$$

where the last inequality follows from (3.24), for $k$ large enough, and the fact that $\psi_{k}(M)=0$. Since $\widehat{v}_{*} \geq-\kappa$ by construction and $\varphi$ is bounded, this implies that the sequence $\left(t_{k}, x_{k}, p_{k}\right)_{k \geq 1}$ 
is bounded, and therefore converges to some $\left(t_{*}, x_{*}, p_{*}\right)$ up to a subsequence. Clearly, $p_{*}=M$, since otherwise we would have $k\left(M-p_{k}\right) \rightarrow \infty$. By definition of $\left(t_{0}, x_{0}\right)$, this implies that

$$
\begin{aligned}
& \left(\widehat{v}_{*}(\cdot, M)-\varphi\right)\left(t_{0}, x_{0}\right) \\
& \geq \liminf _{k \rightarrow \infty}\left(\widehat{v}_{*}-\varphi_{k}\right)\left(t_{k}, x_{k}, p_{k}\right) \\
& \geq\left(\widehat{v}_{*}(\cdot, M)-\varphi\right)\left(t_{*}, x_{*}\right)+\left(\left|x_{*}-x_{0}\right|^{4}+\left(t_{*}-t_{0}\right)^{2}+\liminf _{k \rightarrow \infty} \frac{\rho k}{2(e-1)}\left(M-p_{k}\right)\right) \\
& \geq\left(\widehat{v}_{*}(\cdot, M)-\varphi\right)\left(t_{0}, x_{0}\right)+\left(\left|x_{*}-x_{0}\right|^{4}+\left(t_{*}-t_{0}\right)^{2}+\liminf _{k \rightarrow \infty} \frac{\rho k}{2(e-1)}\left(M-p_{k}\right)\right) .
\end{aligned}
$$

This shows that, after possibly passing to a subsequence,

$$
\left(t_{k}, x_{k}, p_{k}\right) \rightarrow\left(t_{0}, x_{0}, M\right), \quad k\left(M-p_{k}\right) \rightarrow 0, \quad \text { and } \quad \widehat{v}_{*}\left(t_{k}, x_{k}, p_{k}\right) \rightarrow \widehat{v}_{*}\left(t_{0}, x_{0}, M\right) .
$$

b. We now go on with the arguments of [3], up to a non trivial adaptation required by the non-local parts of the operator. In order to prove (3.17), we assume

$$
\widehat{v}_{*}\left(t_{0}, x_{0}, M\right)-v_{*}\left(t_{0}, x_{0}\right)<0
$$

and we intend to prove that

$$
H^{*} \varphi\left(t_{0}, x_{0}\right) \geq 0
$$

By (3.27) and the lower semicontinuity of $\widehat{v}_{*}$, it follows from (3.28) that the sequence $\left(t_{k}, x_{k}, p_{k}\right)_{k>1}$ of minimizers of the difference $\widehat{v}_{*}-\varphi_{k}$ satisfies $\varphi_{k}\left(t_{k}, x_{k}, p_{k}\right)-v_{*}\left(t_{k}, x_{k}\right)<0$, after possibly passing to a subsequence. By Corollary 3.1 together with the result of step 1, Remark 2.5 and Assumption 3.7, we then deduce that

$$
\widehat{H}^{*}\left(t_{k}, x_{k}, p_{k}, \varphi_{k}, \partial_{t} \varphi_{k}, D \varphi_{k}, D^{2} \varphi_{k}, \widehat{v}_{*}\right) \geq 0 \quad \text { for every } \quad k>1 .
$$

Now observe that, by (3.27), and the definition of $\varphi_{k}$ :

$$
\begin{aligned}
\left(\partial_{t} \varphi_{k}, D_{x} \varphi_{k}, D_{x x}^{2} \varphi_{k}\right)\left(t_{k}, x_{k}, p_{k}\right) & \rightarrow\left(\partial_{t} \varphi, D_{x} \varphi, D_{x x}^{2} \varphi\right)\left(t_{0}, x_{0}\right) \quad \text { as } k \rightarrow \infty \\
\left(D_{p} \varphi_{k}, D_{x p}^{2} \varphi_{k}, D_{p p}^{2} \varphi_{k}\right)\left(t_{k}, x_{k}, p_{k}\right) & =\left(-\psi_{k}^{\prime}\left(p_{k}\right), 0,-\psi_{k}^{\prime \prime}\left(p_{k}\right)\right) \quad \text { for every } k>1 .
\end{aligned}
$$

By definition of $\widehat{H}^{*}$, we can find sequences $\left(\varepsilon_{k}\right)_{k>1},\left(\widehat{x}_{k}^{0}\right)_{k>1},\left(y_{k}\right)_{k>1},\left(q_{k}\right)_{k>1},\left(A_{k}\right)_{k>1}$ such that $\varepsilon_{k}>0, \widehat{x}_{k}^{0}=\left(x_{k}^{0}, p_{k}^{0}\right) \in \mathbf{X} \times[m, M], y_{k} \geq-\kappa, q_{k}=\left(q_{k}^{x}, q_{k}^{p}\right) \in \mathbb{R}^{d} \times \mathbb{R}, A_{k}$ is a symmetric matrix of $\mathbb{S}^{d+1}$, with rows $\left(A_{k}^{x x}, A_{k}^{x p}\right) \in \mathbb{S}^{d} \times \mathbb{R}^{d}$ and $\left(A_{k}^{x p T}, A_{k}^{p p}\right) \in \mathbb{R}^{d} \times \mathbb{R}$,

$$
\varepsilon_{k} \rightarrow 0, \quad \widehat{x}_{k}^{0} \rightarrow\left(x_{0}, M\right) \quad \text { and } \quad\left|\left(y_{k}, q_{k}, A_{k}\right)-\left(\varphi_{k}, D \varphi_{k}, D^{2} \varphi_{k}\right)\left(t_{k}, x_{k}, p_{k}\right)\right| \leq k^{-1},
$$

where $\left(t_{k}, \widehat{x}_{k}^{0}\right)$ belongs to a compact neighborhood of $\left(t_{0}, x_{0}, M\right)$, and

$$
\widehat{H}_{\varepsilon_{k},-k^{-1}}\left(t_{k}, \widehat{x}_{k}^{0}, y_{k}, \partial_{t} \varphi\left(t_{0}, x_{0}\right), q_{k}, A_{k}, \widehat{v}_{*}\right) \geq-k^{-1} .
$$

By the definition of $\widehat{H}_{\varepsilon_{k},-k^{-1}}$, we may find a sequence $\left(u_{k}, \alpha_{k}, \pi_{k}\right) \in \widehat{\mathcal{N}}_{\varepsilon_{k},-2 k^{-1}}\left(t_{k}, \widehat{x}_{k}^{0}, y_{k}, q_{k}, \widehat{v}_{*}\right)$ such that

$$
\begin{aligned}
-\partial_{t} \varphi\left(t_{0}, x_{0}\right) & +\mu_{Y}\left(x_{k}^{0}, y_{k}, u_{k}\right)-\mu_{X}\left(x_{k}^{0}, u_{k}\right) \cdot q_{k}^{x}-\frac{1}{2} \operatorname{Tr}\left[\sigma_{X} \sigma_{X}^{T}\left(x_{k}^{0}, u_{k}\right) A_{k}^{x x}\right] \\
& \geq-2 k^{-1}+\frac{1}{2}\left|\alpha_{k}\right|^{2} A_{k}^{p p}+\sigma_{X}^{T}\left(x_{k}^{0}, u_{k}\right) A_{k}^{x p} \cdot \alpha_{k}-\int_{E} \pi_{k}(e) \lambda(d e) q_{k}^{p}
\end{aligned}
$$

and

$$
\begin{gathered}
\beta_{Y}\left(x_{k}^{0}, y_{k}, u_{k}(e), e\right)-\widehat{v}_{*}\left(t_{k}, x_{k}^{0}+\beta_{X}\left(x_{k}^{0}, u_{k}(e), e\right), p_{k}^{0}+\pi_{k}(e)\right)+y_{k} \geq-2 k^{-1} \\
\text { for } \lambda \text {-a.e. } e \in E .
\end{gathered}
$$


Recalling (3.14), we observe that $\left(u_{k}, \alpha_{k}, \pi_{k}\right) \in \widehat{\mathcal{N}}_{\varepsilon_{k},-2 k^{-1}}\left(t_{k}, \widehat{x}_{k}, y_{k}, q_{k}, \widehat{v}_{*}\right)$ implies that $u_{k} \in$ $\widetilde{\mathcal{N}}_{\varepsilon_{k}+\left|q_{k}^{p} \alpha_{k}\right|}\left(x_{k}^{0}, y_{k}, q_{k}^{x}\right)$.

We deduce then from Assumption 3.6 and (3.33) that, for some constant $C>0$, (which may change from line to line but does not depend on $k$ or $\rho$ ),

$$
\begin{aligned}
C\left(1+\left|q_{k}^{p} \alpha_{k}\right|^{2}\right) & \geq \frac{1}{2}\left|\alpha_{k}\right|^{2} A_{k}^{p p}+\sigma_{X}^{T}\left(x_{k}^{0}, u_{k}\right) A_{k}^{x p} \cdot \alpha_{k}-\int_{E} \pi_{k}(e) \lambda(d e) q_{k}^{p} \\
& \geq \frac{1}{2}\left|\alpha_{k}\right|^{2} A_{k}^{p p}-C\left|A_{k}^{x p}\right|\left|\alpha_{k}\right|-\int_{E} \pi_{k}(e) \lambda(d e) q_{k}^{p}
\end{aligned}
$$

where we used the condition that $\sup _{u \in U}\left|\sigma_{X}(\cdot, u)\right|$ is locally bounded. From (3.24), (3.25), (3.26), (3.27), (3.30) and (3.31), it follows that

$$
A_{k}^{p p} \rightarrow+\infty, \quad A_{k}^{x p} \rightarrow 0, \quad q_{k}^{p} \rightarrow+\infty \quad \text { and } \quad \frac{\left(q_{k}^{p}\right)^{2}}{\left|A_{k}^{p p}\right|} \rightarrow \rho \text { as } k \rightarrow \infty .
$$

Recall from (3.5) that

$$
\pi_{k} \leq M-p_{k} \quad \lambda \text {-a.e., }
$$

where $p_{k} \in[m, M]$. We may hence consider that $\left(\pi_{k}\right)_{k \geq 1}$ is bounded from above, so that, by (3.35) and the fact that $q_{k}^{p}, A_{k}^{p p}>0$

$$
C\left(\frac{1}{A_{k}^{p p}}+\frac{\left|q_{k}^{p}\right|^{2}}{A_{k}^{p p}}\left|\alpha_{k}\right|^{2}\right) \geq \frac{1}{2}\left|\alpha_{k}\right|^{2}-C \frac{\left|A_{k}^{x p}\right|^{2}}{A_{k}^{p p}}\left|\alpha_{k}\right|-C \frac{q_{k}^{p}}{A_{k}^{p p}} .
$$

Hence, (3.36) leads to

$$
0 \geq \limsup _{k \rightarrow \infty}\left(\left(\frac{1}{2}-C \rho\right)\left|\alpha_{k}\right|^{2}-C \frac{\left|A_{k}^{x p}\right|^{2}}{A_{k}^{p p}}\left|\alpha_{k}\right|\right) .
$$

Taking $\rho$ small enough implies that

$$
\left|\alpha_{k}\right| \underset{k \rightarrow \infty}{\rightarrow} 0 .
$$

Moreover, since $k\left(M-p_{k}\right) \rightarrow 0$, see $(3.27)$, there exists $\epsilon_{k} \downarrow 0$ such that $k\left(M-p_{k}\right) \leq \epsilon_{k}$. Recalling (3.37), this implies that $\pi_{k} \leq \frac{\epsilon_{k}}{k}$, so that, by (3.24),

$$
q_{k}^{p}\left(\pi_{k}(e)\right)^{+} \rightarrow 0 \quad \text { as } \quad k \rightarrow \infty \quad \text { for all } e \in E .
$$

Recalling the fact that $\lambda(E)<\infty$ and that $q_{k}^{p}>0$, the above inequalities lead to

$$
\left(\int_{E} \pi_{k}(e) \lambda(d e) q_{k}^{p}\right)^{+} \rightarrow 0
$$

Also recall that $\frac{\left|q_{k}^{p}\right|^{2}}{A_{k}^{p p}} \rightarrow \rho$, see (3.36), which combined with (3.35), (3.36), (3.38) and (3.40), implies that

or equivalently

$$
C\left(1+\rho A_{k}^{p p}\left|\alpha_{k}\right|^{2}\right) \geq \frac{1}{2}\left|\alpha_{k}\right|^{2} A_{k}^{p p}+\left(\int_{E} \pi_{k}(e) \lambda(d e) q_{k}^{p}\right)^{-}
$$

$$
C\left(1+\left|q_{k}^{p}\right|^{2}\left|\alpha_{k}\right|^{2}\right) \geq \frac{1}{2}\left|\alpha_{k}\right|^{2} \frac{\left|q_{k}^{p}\right|^{2}}{\rho}+\left(\int_{E} \pi_{k}(e) \lambda(d e) q_{k}^{p}\right)^{-}
$$

for some $\rho>0$. Taking $\rho$ small enough leads to

$$
\left|A_{k}^{p p}\right|\left|\alpha_{k}\right|^{2} \leq C, \quad\left|q_{k}^{p}\right|^{2}\left|\alpha_{k}\right|^{2} \leq C \rho \quad \text { and } \quad C+C \rho \geq\left(\int_{E} \pi_{k}(e) \lambda(d e) q_{k}^{p}\right)^{-} .
$$


We then deduce from the right hand side bound of (3.24) and (3.31) that

$$
0 \geq \limsup _{k \rightarrow+\infty}\left(\int_{E} \pi_{k}(e) \lambda(d e)\right)^{-}
$$

Combined with (3.39), this shows that

$$
\int_{E} \pi_{k}(e) \lambda(d e) \rightarrow 0 \quad \text { and } \quad \pi_{k}(e) \rightarrow 0 \quad \text { for } \lambda \text {-a.e. } e \in E \text {. }
$$

c. We now return to (3.33) and the middle inequality in (3.41) to deduce that

$$
\begin{gathered}
-\partial_{t} \varphi\left(t_{0}, x_{0}\right)+\mu_{Y}\left(x_{k}^{0}, y_{k}, u_{k}\right)-\mu_{X}\left(x_{k}^{0}, u_{k}\right) \cdot q_{k}^{x}-\frac{1}{2} \operatorname{Tr}\left[\sigma_{X} \sigma_{X}^{T}\left(x_{k}^{0}, u_{k}\right) A_{k}^{x x}\right] \\
\geq-2 k^{-1}+\sigma_{X}^{T}\left(x_{k}^{0}, u_{k}\right) A_{k}^{x p} \cdot \alpha_{k}-\left(\int_{E} \pi_{k}(e) \lambda(d e) q_{k}^{p}\right)^{+}
\end{gathered}
$$

and

$$
u_{k} \in \widetilde{\mathcal{N}}_{\varepsilon_{k}+\sqrt{C \rho}}\left(x_{k}^{0}, y_{k}, q_{k}^{x}\right) .
$$

since $A_{k}^{p p}>0$.

Consider now (3.34), i.e.

$\beta_{Y}\left(x_{k}^{0}, y_{k}, u_{k}(e), e\right)-\widehat{v}_{*}\left(t_{k}, x_{k}^{0}+\beta_{X}\left(x_{k}^{0}, u_{k}(e), e\right), p_{k}^{0}+\pi_{k}(e)\right)+y_{k} \geq-2 k^{-1} \quad$ for $\lambda$-a.e. $e \in E$,

Using the upper semi-continuity of $-\widehat{v}_{*}$, the fact that $\beta_{Y}$ is continuous, (3.42), together with $p_{k}^{0} \rightarrow M$ as $k \rightarrow \infty$, we obtain

$$
\beta_{Y}\left(x_{k}^{0}, y_{k}, u_{k}(e), e\right)-\widehat{v}_{*}\left(t_{k}, x_{k}^{0}+\beta_{X}\left(x_{k}^{0}, u_{k}(e), e\right), M\right)+y_{k} \geq-2 k^{-1}-\vartheta_{k}^{e}
$$

for $k$ large enough and for $\lambda$-a.e. $e \in E$,

with $\vartheta_{k}^{e} \geq 0$ such that $\vartheta_{k}^{e} \rightarrow 0$ as $k \rightarrow \infty$ for all $e \in E$.

We now use Assumption 3.4 to deduce that there exists $\vartheta_{k}>0$ with $\vartheta_{k} \rightarrow 0$ as $k \rightarrow \infty$ such that, for all $e \in E$ and $k$ large enough,

$$
\beta_{Y}\left(x_{k}^{0}, y_{k}, u_{k}(e), e\right)-\widehat{v}_{*}\left(t_{k}, x_{k}^{0}+\beta_{X}\left(x_{k}^{0}, u_{k}(e), e\right), M\right)+y_{k} \geq-2 k^{-1}-\vartheta_{k} .
$$

By combining (3.43) (3.44) and (3.46), we finally obtain

$$
\begin{aligned}
H_{\varepsilon_{k}+\sqrt{C \rho},-2 k^{-1}-\vartheta_{k}} & \left(t_{k}, x_{k}^{0}, y_{k}, \partial_{t} \varphi\left(t_{0}, x_{0}\right), q_{k}^{x}, A_{k}^{x x}, \widehat{v}_{*}(\cdot, M)\right) \\
& \geq-2 k^{-1}-\left(\sigma_{X}^{T}\left(x_{k}^{0}, u_{k}\right) A_{k}^{x p} \cdot \alpha_{k}\right)^{-}-\left(\int_{E} \pi_{k}(e) \lambda(d e) q_{k}^{p}\right)^{+},
\end{aligned}
$$

and we deduce the required result (3.29) by sending $k \rightarrow \infty$ and then $\rho \rightarrow 0$, and recalling that $\left(\left|\alpha_{k}\right|, A_{k}^{x p},\left(\int_{E} \pi_{k}(e) \lambda(d e) q_{k}^{p}\right)^{+}\right) \rightarrow 0$, that $\sigma_{X}$ is locally bounded uniformly in the control $u$ and that $\widehat{v}_{*} \geq \varphi$.

$3^{\text {rd }}$ step: It remains to prove $(3.18)$. The fact that $\widehat{v}_{*}(T, \cdot, M)$ is a viscosity supersolution

$$
\max \left\{\widehat{v}_{*}(T, \cdot, M)-v_{*}(T, \cdot), \delta^{*} \widehat{v}_{*}(T, \cdot, M)\right\} \geq 0
$$

is deduced from (3.22) of the previous step by using the same arguments as in the proof of Lemma 2.1 in Section 2.3.2. It remains to show that $\widehat{v}_{*}(T, \cdot, M)$ is a viscosity supersolution of

$$
\max \left\{\widehat{v}_{*}(T, \cdot, M)-v_{*}(T, \cdot),\left(\widehat{v}_{*}(T, \cdot, M)-j_{*}\right) \mathbb{1}_{\left\{H^{*} \widehat{v}_{*}(T, \cdot, M)<\infty\right\}}\right\} \geq 0 .
$$

By combining the arguments of step 1 with those of Section 2.3.2, we first show that for any smooth function $\widehat{\varphi}$ on $\mathbf{X} \times[m, M]$ and $x_{0} \in \mathbf{X}$ such that 


$$
\text { (strict) } \min _{\mathbf{X} \times[m, M]}\left(\widehat{v}_{*}(T, \cdot)-\widehat{\varphi}\right)=\left(\widehat{v}_{*}(T, \cdot)-\widehat{\varphi}\right)\left(x_{0}, M\right)=0,
$$

we have

$$
\max \left\{\widehat{\varphi}\left(x_{0}, M\right)-v_{*}\left(T, x_{0}\right),\left(\widehat{\varphi}\left(x_{0}, M\right)-\widehat{g}_{*}\left(x_{0}\right)\right) \mathbb{1}_{\left\{\widehat{H}^{*} \widehat{\varphi}\left(x_{0}, M\right)<\infty\right\}}\right\} \geq 0 .
$$

We then consider a smooth function $\varphi$ on $\mathbf{X}$ and $x_{0} \in \mathbf{X}$ such that

$$
\text { (strict) } \min _{\mathbf{X}}\left(\widehat{v}_{*}(T, \cdot, M)-\varphi\right)=\left(\widehat{v}_{*}(T, \cdot, M)-\varphi\right)\left(x_{0}\right)=0
$$

and

$$
\varphi\left(x_{0}\right)<\widehat{v}\left(T, x_{0}\right)
$$

and we assume that

$$
H^{*} \varphi\left(T, x_{0}\right)<\infty .
$$

We next follow the construction of step 2 of the modified test functions

$$
\varphi_{k}:=\varphi(x)-\left(\left|x-x_{0}\right|^{4}+\psi_{k}(p)\right),
$$

where $\psi_{k}$ is defined in (3.23). As in the above step 2, one can prove that the difference $\widehat{v}_{*}(T, \cdot)-$ $\varphi_{k}$ has a local minimizer $\widehat{x}_{k}=\left(x_{k}, p_{k}\right)$ satisfying all estimates derived in the above step 2 (forgetting about the $t$ variable). In particular, since $H^{*} \varphi_{k}\left(x_{k}\right) \leq C$ for some constant $C>$ 0 independent of $k$, recall (3.49), we deduce from the same estimates than in step 2 that $\widehat{H}^{*} \varphi_{k}\left(\widehat{x}_{k}\right) \leq 2 C$ for all large $k$. It then follows from Corollary 3.2, (3.47) and (3.49) that $\widehat{v}_{*}\left(T, \widehat{x}_{k}\right) \geq \widehat{g}_{*}\left(\widehat{x}_{k}\right)$. Sending $k \rightarrow \infty$, this provides $\widehat{v}_{*}\left(T, x_{0}, M\right) \geq \widehat{g}_{*}\left(x_{0}, M\right)$, and the proof is completed by observing that $\widehat{g}_{*}\left(x_{0}, M\right)=j_{*}\left(x_{0}\right)$, by definition of $j$.

\subsubsection{The endpoint $p=m$, finite}

We organize the proof in four steps. As in the previous section, steps 1, 2 and 3 focus on $t<T$ while step 4 concentrates on $t=T$. Steps 1 and 4 are similar to arguments used in [3]. The main difference comes from steps 2 and 3.

$1^{\text {st }}$ step: We first show that for any smooth function $\widehat{\varphi}$ on $[0, T) \times \mathbf{X} \times[m, M]$ and $\left(t_{1}, x_{1}\right) \in$

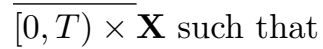

$$
\text { (strict) } \max _{[0, T) \times \mathbf{X} \times[m, M]}\left(\widehat{v}^{*}-\widehat{\varphi}\right)=\left(\widehat{v}^{*}-\widehat{\varphi}\right)\left(t_{1}, x_{1}, m\right)=0,
$$

we have

$$
\min \left\{\widehat{v}^{*}+\kappa, \widehat{H}_{*} \widehat{\varphi}\right\}\left(t_{1}, x_{1}, m\right) \leq 0
$$

The proof is very similar to that of Sections 2.3.3 up to the modification explained in the proof of Corollary 3.2 , and the fact that we have to handle the state constraint $p=m$. For completeness, we report here the entire argument. Assume to the contrary that

$$
4 \eta:=\min \left\{\widehat{v}^{*}+\kappa, \widehat{H}_{*} \widehat{\varphi}\right\}\left(t_{1}, x_{1}, m\right)>0
$$

i.e., for some $\varepsilon>0$, and after possibly changing $\eta>0$,

$$
\begin{aligned}
& \min \left\{\widehat{\varphi}_{\iota}(t, \widehat{x})+\kappa, \mu_{Y}(x, y, \widehat{u})-\widehat{\mathcal{L}}^{\widehat{u}} \widehat{\varphi}_{\iota}(t, \widehat{x})\right\} \geq 2 \eta \\
& \text { for some } \widehat{u}=(u, \alpha, \pi) \in \widehat{\mathcal{N}}_{0, \eta}\left(t, \widehat{x}, y, D \widehat{\varphi}_{\iota}(t, \widehat{x}), \widehat{\varphi}_{\iota}\right) \\
& \text { for all } \quad(t, \widehat{x}, y) \in[0, T) \times \widehat{\mathbf{X}} \times \mathbb{R} \\
& \text { s.t. } \quad(t, \widehat{x}) \in B_{\varepsilon}\left(t_{1}, x_{1}\right) \times[m, m+\varepsilon],\left|y-\widehat{\varphi}_{\iota}(t, \widehat{x})\right| \leq \varepsilon,
\end{aligned}
$$


where $\widehat{\varphi}_{\iota}(t, \widehat{x}):=\widehat{\varphi}(t, \widehat{x})+\iota\left(\left|x-x_{1}\right|^{4}+|p-m|^{4}\right)$ with $\iota$ small enough. Then, Assumption 3.2 and Assumption 3.4 imply that

$$
\begin{gathered}
\min \left\{\widehat{\varphi}_{\iota}(t, \widehat{x})+\kappa, \mu_{Y}\left(x, y, \widehat{\nu}\left(t, \widehat{x}, y, D \widehat{\varphi}_{\iota}(t, \widehat{x})\right)\right)-\widehat{\mathcal{L}}^{\widehat{\nu}\left(t, \widehat{x}, y, D \widehat{\varphi}_{\iota}(t, \widehat{x})\right)} \widehat{\varphi}_{\iota}(t, \widehat{x}),\right. \\
\left.\min _{e \in E} \widehat{\mathcal{G}}^{\widehat{\nu}\left(t, \widehat{x}, y, D \widehat{\varphi}_{\iota}(t, \widehat{x})\right), e} \widehat{\varphi}_{\iota}(t, \widehat{x})\right\} \geq \eta \\
\text { for }(t, \widehat{x}, y) \in[0, T] \times \widehat{\mathbf{X}} \times \mathbb{R} \text { s.t. } \\
(t, \widehat{x}) \in B_{\varepsilon}\left(t_{1}, x_{1}\right) \times[m, m+\varepsilon] \quad \text { and }\left|y-\widehat{\varphi}_{\iota}(t, \widehat{x})\right| \leq \varepsilon,
\end{gathered}
$$

where $\widehat{\nu}$ is a locally Lipschitz map satisfying

$$
\widehat{\nu}\left(t, \widehat{x}, y, D \widehat{\varphi}_{\iota}(t, \widehat{x})\right) \in \widehat{\mathcal{N}}_{0, \eta}\left(t, \widehat{x}, y, D \widehat{\varphi}_{\iota}(t, \widehat{x}), \widehat{\varphi}_{\iota}\right) \quad \text { on } \quad B_{\varepsilon}\left(t_{1}, x_{1}\right) \times[m, m+\varepsilon] .
$$

Observe that, since $\left(t_{1}, x_{1}, m\right)$ is a strict maximizer in (3.51), we have

$$
-\xi:=-\left(\zeta \wedge \iota \varepsilon^{4}\right):=\max _{D}\left(\widehat{v}_{*}-\widehat{\varphi}_{\iota}\right)<0,
$$

where

$$
D:=\left(\left\{t_{1}+\varepsilon\right\} \times \bar{B}_{\varepsilon}\left(x_{1}\right) \times[m, m+\varepsilon]\right) \cup\left(\left[t_{1}, t_{1}+\varepsilon\right) \times\left(B_{\varepsilon}\left(x_{1}\right) \times[m, m+\varepsilon)\right)^{c}\right) .
$$

Also, we deduce from (3.53) and the fact that $\widehat{v}(\cdot, m)=-\kappa$ by definition, that

$$
0>-\eta \geq \max _{B_{\varepsilon}\left(t_{1}, x_{1}\right)}(\widehat{v}-\widehat{\varphi})(\cdot, m) .
$$

By following the arguments in step 2 of Section 2.3.3, we see that (3.54), (3.55), (3.56) and (3.57) lead to a contradiction of (GDP2).

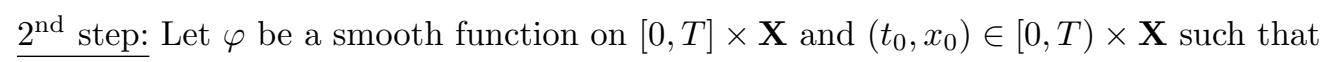

$$
\text { (strict) } \max _{[0, T) \times \mathbf{X}}\left(\widehat{v}^{*}(\cdot, m)-\varphi\right)=\left(\widehat{v}^{*}(\cdot, m)-\varphi\right)\left(t_{0}, x_{0}\right)=0 .
$$

By definition, we have $\widehat{v}^{*}\left(t_{0}, x_{0}, m\right) \geq-\kappa$. Let us assume that

$$
\widehat{v}^{*}\left(t_{0}, x_{0}, m\right)+\kappa=: 4 \eta>0,
$$

and work towards a contradiction. Define the function $\psi_{k}$ as in (3.23) with $m$ in place $M$ :

$$
\psi_{k}(p):=\rho k \int_{m}^{p} \frac{e^{2 k m}}{e^{k(r+m)}-e^{2 k m+1}} d r, \quad k>0,
$$

and

$$
\varphi_{k}(t, x, p):=\varphi(t, x)+\left(\left|x-x_{0}\right|^{4}+\left(t-t_{0}\right)^{2}+\psi_{k}(p)\right) .
$$

Arguing as in step 2 of the section, we see that the difference $\widehat{v}^{*}-\varphi_{k}$ has a local maximizer $\left(t_{k}, x_{k}, p_{k}\right)$ on $\overline{([0, T] \times \mathbf{X} \times[m, M])}$ satisfying

$$
\left(t_{k}, x_{k}, p_{k}\right) \rightarrow\left(t_{0}, x_{0}, m\right), \quad k\left(p_{k}-m\right) \rightarrow 0 \quad \text { and } \quad \widehat{v}^{*}\left(t_{k}, x_{k}, p_{k}\right) \rightarrow \widehat{v}^{*}\left(t_{0}, x_{0}, m\right),
$$

so that

$$
\begin{gathered}
\left(\partial_{t} \varphi_{k}, D_{x} \varphi_{k}, D_{x x}^{2} \varphi_{k}\right)\left(t_{k}, x_{k}, p_{k}\right) \rightarrow\left(\partial_{t} \varphi, D_{x} \varphi, D_{x x}^{2} \varphi\right)\left(t_{0}, x_{0}\right) \quad \text { as } \quad k \rightarrow \infty \\
\left(D_{p} \varphi_{k}, D_{x p}^{2} \varphi_{k}, D_{p p}^{2} \varphi_{k}\right)\left(t_{k}, x_{k}, p_{k}\right)=\left(\psi_{k}^{\prime}\left(p_{k}\right), 0, \psi_{k}^{\prime \prime}\left(p_{k}\right)\right) .
\end{gathered}
$$

Since $\widehat{v}^{*}\left(t_{0}, x_{0}, m\right)>-\kappa$, we have $\widehat{v}^{*}\left(t_{k}, x_{k}, p_{k}\right)>-\kappa$ for all $k$, after possibly passing to a subsequence. Then, it follows from Corollary 3.1, step 1 and the arguments of Remark 2.5 under Assumption 3.7, that 


$$
\widehat{H}_{*}\left(\cdot, \varphi_{k}, \partial_{t} \varphi_{k}, D \varphi_{k}, D^{2} \varphi_{k}, \widehat{v}^{*}\right)\left(t_{k}, x_{k}, p_{k}\right) \leq 0 \text { for } k>1 .
$$

By the definition of $\widehat{H}_{*}$, we deduce that there exist sequences $\left(\varepsilon_{k}\right)_{k \geq 1},\left(\widehat{x}_{k}\right)_{k \geq 1},\left(y_{k}\right)_{k \geq 1},\left(q_{k}\right)_{k \geq 1}$ and $\left(A_{k}\right)_{k \geq 1}$ such that $\varepsilon_{k}>0, \widehat{x}_{k}^{0}=\left(x_{k}^{0}, p_{k}^{0}\right) \in \mathbf{X} \times[m, M], y_{k} \geq-\kappa, q_{k}=\left(q_{k}^{x}, q_{k}^{p}\right) \in \mathbb{R}^{d} \times \mathbb{R}$, and $A_{k} \in \mathbb{S}^{d+1}$ with rows $\left(A_{k}^{x x}, A_{k}^{x p}\right) \in \mathbb{S}^{d} \times \mathbb{R}^{d}$ and $\left(A_{k}^{x p T}, A_{k}^{p p}\right) \in \mathbb{R}^{d} \times \mathbb{R}$ satisfying

$$
\varepsilon_{k} \rightarrow 0, \quad \widehat{x}_{k}^{0} \rightarrow\left(x_{0}, m\right), \quad \text { and } \quad\left|\left(y_{k}, q_{k}, A_{k}\right)-\left(\varphi_{k}, D \varphi_{k}, D^{2} \varphi_{k}\right)\left(t_{k}, x_{k}, p_{k}\right)\right| \leq k^{-1}
$$

for which

$$
\widehat{H}_{\varepsilon_{k}, k^{-1}}\left(t_{k}, \widehat{x}_{k}, y_{k}, \partial_{t} \varphi\left(t_{0}, x_{0}\right), q_{k}, A_{k}, \widehat{v}^{*}\right) \leq k^{-1} .
$$

Fix $u \in U, \pi=0$ and set $\alpha_{k}:=N^{u}\left(x_{k}^{0}, y_{k}, q_{k}^{x}\right) / q_{k}^{p}$. Since $\pi=0$, it follows from (3.60) together with (3.5), (3.6) and Assumption 3.4 that either $\left(u, \alpha_{k}, \pi\right) \in \widehat{\mathcal{N}}_{\varepsilon_{k}, k^{-1}}\left(t, \widehat{x}_{k}, y_{k}, q_{k}, \widehat{v}^{*}\right)$ and then

$$
\begin{aligned}
\mu_{Y} & \left(x_{k}^{0}, y_{k}, u\right)-\partial_{t} \varphi\left(t_{0}, x_{0}\right)-\mu_{X}\left(x_{k}^{0}, u\right) \cdot q_{k}^{x} \\
& -\frac{1}{2}\left(\operatorname{Tr}\left[\sigma_{X} \sigma_{X}^{T}\left(x_{k}^{0}, u\right) A_{k}^{x x}\right]+|\alpha|^{2} A_{k}^{p p}+2 \sigma_{X}^{T}\left(x_{k}^{0}, u\right) A_{k}^{x p} \cdot \alpha\right) \leq k^{-1}
\end{aligned}
$$

or

$$
\beta_{Y}\left(x_{k}^{0}, y_{k}, u\left(e_{k}\right), e_{k}\right)-\widehat{v}^{*}\left(t_{k}, x_{k}^{0}+\beta_{X}\left(x_{k}^{0}, u\left(e_{k}\right), e_{k}\right), p_{k}^{0}\right)+y_{k} \leq k^{-1},
$$

for some sequence $\left(e_{k}\right)_{k \geq 1} \subseteq E$. Using the same kind of arguments as in step 2 of the previous section leads to

$$
A_{k}^{p p}<0, \quad q_{k}^{p}<0 \text { for large } k, \quad \lim _{k \rightarrow \infty} A_{k}^{x p}=0 \quad \text { and } \quad \lim _{k \rightarrow \infty} \frac{\left(q_{k}^{p}\right)^{2}}{\left|A_{k}^{p p}\right|}=\rho .
$$

Consider first the case where (3.61) holds along a subsequence. Using (3.61) and (3.63), we then deduce that

$$
\left|A_{k}^{p p}\right|\left|\alpha_{k}\right|^{2}=\frac{\left|A_{k}^{p p}\right|}{\left(q_{k}^{p}\right)^{2}}\left|N^{u}\left(x_{k}^{0}, y_{k}, q_{k}^{x}\right)\right|^{2} \leq C,
$$

for some $C>0$ independent of $k$ and $\rho$. Sending $k \rightarrow \infty$ in the above inequality, we then deduce from (3.59) and (3.63) that

$$
\rho^{-1}\left|N^{u}\left(x_{0}, \varphi\left(t_{0}, x_{0}\right), D \varphi\left(t_{0}, x_{0}\right)\right)\right|^{2} \leq C .
$$

Since $\rho>0$ can be chosen arbitrarily close to 0 , this shows that $N^{u}\left(x_{0}, \varphi\left(t_{0}, x_{0}\right), D \varphi\left(t_{0}, x_{0}\right)\right)=$ 0 , and the arbitrariness of $u \in U$ is in contradiction with Assumption 3.5. This contradicts (3.58).

Hence, if (3.58) holds, then (3.62) holds along a subsequence, i.e.

$$
\beta_{Y}\left(x_{k}^{0}, y_{k}, u\left(e_{k}\right), e_{k}\right)-\widehat{v}^{*}\left(t_{k}, x_{k}^{0}+\beta_{X}\left(x_{k}^{0}, u\left(e_{k}\right), e_{k}\right), p_{k}^{0}\right)+y_{k} \leq k^{-1} .
$$

Sending $k \rightarrow \infty$, using the arbitrariness of $u \in U$ and Assumption 3.4 then leads to

$$
\check{G} \widehat{v}^{*}\left(t_{0}, x_{0}, m\right) \leq 0,
$$

where

$$
\check{G} \varphi=\sup _{u \in U} \min _{e \in E}\left\{\beta_{Y}(\cdot, u(e), e)-\varphi\left(\cdot+\beta_{X}(\cdot, u(e), e)\right)+\varphi\right\} .
$$

Hence

$$
\min \left\{\widehat{v}^{*}+\kappa, \check{G} \widehat{v}^{*}\right\}\left(t_{0}, x_{0}, m\right) \leq 0
$$

on $[0, T) \times \mathbf{X}$. 
$3^{\text {rd }}$ step: Now observe that, by standard arguments, for every $(t, x) \in[0, T) \times \mathbf{X}$, we may find a sequence of smooth functions $\left(\varphi^{n}\right)_{n \geq 1}$ such that $\varphi^{n} \downarrow \widehat{v}^{*},\left(t_{n}, x_{n}, p_{n}\right)_{n \geq 1}$ converging towards $(t, x, m)$ and such that $\left(\varphi^{n}-\widehat{v}^{*}\right)$ achieves a maximum at $\left(t_{n}, x_{n}, p_{n}\right)$. We refer to lemma 6.1 in [2] for the approximation argument by continuous functions. The extension to an approximation by smooth functions is straightforward.

It thus follows from step 2 , that $\widehat{v}^{*}(\cdot, m)$ is a classical subsolution of $(3.64)$ on $[0, T) \times \mathbf{X}$. In order to conclude the proof, we now appeal to the following easy lemma.

Lemma 3.1. Assume that H2 holds. Let $w$ be a upper semi-continuous subsolution of

$$
\min \{w+\kappa, \check{G} w\} \leq 0 \text { on } \boldsymbol{X}
$$

such that $w^{+}$satisfies the growth condition (3.13). Then, $w \leq-\kappa$ on $\boldsymbol{X}$.

Applying Lemma 3.1 to $\widehat{v}^{*}\left(t_{0}, \cdot, m\right)$ for an arbitrary $t_{0} \in[0, T)$ then leads to $\widehat{v}^{*}(\cdot, m)=-\kappa$, since $\widehat{v}^{*}(\cdot, m) \geq-\kappa$ and $\widehat{v}^{*-}$ satisfies $(3.13)$ by assumption.

$4^{\text {rd }}$ step: We finally show that $\widehat{v}_{*}(T, \cdot, m)=-\kappa$ on $\mathbf{X}$. Since $\widehat{v}^{*}(t, x, m)=-\kappa$ for $t<T$ and

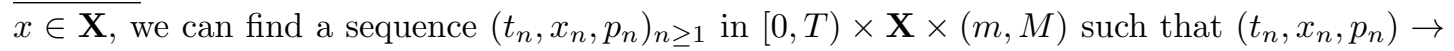
$(T, x, m)$ and $-\kappa \leq \widehat{v}\left(t_{n}, x_{n}, p_{n}\right) \leq-\kappa+\frac{1}{n}$ for all $n \geq 0$. Passing to the limit leads to the required result.

\section{Proof of Lemma 3.1.}

We assume that $\sup _{\mathbf{X}}(w+\kappa)>0$ and work towards a contradiction. It follows from the growth condition (3.13) on $w$, (ii) and (iii) of $\mathbf{H 2}$ that there is some $x_{0} \in \mathbf{X}$ such that

$$
\max _{\mathbf{X}}(w-\Lambda)=(w-\Lambda)\left(x_{0}\right)=: \xi>0 .
$$

By (i) of H2 Assumption 3.4 and (3.66), there exists some $\bar{u} \in U$ such that

$$
\min _{e \in E} \beta_{Y}\left(x_{0}, w\left(x_{0}\right), \bar{u}(e), e\right)-\Lambda\left(x_{0}+\beta_{X}\left(x_{0}, \bar{u}(e), e\right)\right)+\Lambda\left(x_{0}\right)>0 .
$$

Since $w$ is a subsolution on $\mathbf{X}$ of (3.65), we have $\check{G} w\left(x_{0}\right) \leq 0$. Recalling Assumption 3.4, we may then find $\hat{e} \in E$ such that

$$
\beta_{Y}\left(x_{0}, w\left(x_{0}\right), \bar{u}(\hat{e}), \hat{e}\right)-w\left(x_{0}+\beta_{X}\left(x_{0}, \bar{u}(\hat{e}), \hat{e}\right)\right)+w\left(x_{0}\right) \leq 0 .
$$

Combining the last inequality with (3.67) leads to

$$
w\left(x_{0}\right)-\Lambda\left(x_{0}\right)<w\left(x_{0}+\beta_{X}\left(x_{0}, \bar{u}(\hat{e}), \hat{e}\right)\right)-\Lambda\left(x_{0}+\beta_{X}\left(x_{0}, \bar{u}(\hat{e})\right)\right),
$$

which contradicts the definition of $x_{0}$ in (3.66).

\section{References}

[1] G. Barles. Solution de viscosites des inequations d'Hamilton Jacobi. Mathematiques et Applications, 17, 1994.

[2] B. Bouchard. Stochastic targets with mixed diffusion processes. Stochastic processes and their Applications, 101:273-302, 2002.

[3] B. Bouchard, R. Elie, and N. Touzi. Stochastic target problems with controlled loss. SIAM Journal on Control and Optimization, 48:3123, 2009.

[4] B. Bouchard and T.N. Vu. The obstacle version of the geometric dynamic programming principle: Application to the pricing of american options under constraints. Applied Mathematics and Optimization. 
[5] W.H. Fleming and H.M. Soner. Controlled markov processes and viscosity solutions. Mathematics and Applications, 25, 1993.

[6] H. Follmer and P. Leukert. Quantile hedging. Finance and Stochastics, 3:251-273, 1999.

[7] H.M. Soner. Optimal control with state-space constraint I. SIAM Journal on Control and Optimization, 24:552-561, 1986.

[8] H.M. Soner. Optimal control with state-space constraint II. SIAM Journal on Control and Optimization, 24:1110-1122, 1986.

[9] H.M. Soner and N. Touzi. Dynamic programming for stochastic target problems and geometric flows. Journal of the European Mathematical Society, 4:201-236, 2002.

[10] H.M. Soner and N. Touzi. Stochastic target problems, dynamic programming and viscosity solutions. SIAM Journal on Control and Optimization, 41:404-424, 2002. 